\title{
THE INCOMPRESSIBLE EULER LIMIT OF THE BOLTZMANN EQUATION WITH ACCOMMODATION BOUNDARY CONDITION*
}

\author{
CLAUDE BARDOS ${ }^{\dagger}$, FRANÇOIS GOLSE ${ }^{\ddagger}$, AND LIONEL PAILLARD ${ }^{\S}$
}

To Dave Levermore on his 60th birthday with friendship and appreciation

\begin{abstract}
The convergence of solutions of the Navier-Stokes Equations, set in a domain with boundary, to solutions of the Euler Equations in the large Reynolds number limit is a challenging open problem in both 2 and 3 space dimensions. In particular it is distinct from the question of existence in the large of a smooth solution of the initial-boundary value problem for the Euler Equations. The present paper proposes three results in that direction. First, if the solutions of the Navier-Stokes Equations satisfy a slip boundary condition with vanishing slip coefficient in the large Reynolds number limit, we show by an energy method that they converge to the classical solution of the Euler Equations on its time interval of existence. Next we show that the incompressible NavierStokes limit of the Boltzmann Equation with Maxwell's accommodation condition at the boundary is governed by the Navier-Stokes Equations with slip boundary condition, and we express the slip coefficient at the fluid level in terms of the accommodation parameter at the kinetic level. This second result is formal, in the style of [Bardos-Golse-Levermore, J. Stat. Phys., 63, 323-344, 1991]. Finally, we establish the incompressible Euler limit of the Boltzmann Equation set in a domain with boundary with Maxwell's accommodation condition assuming that the accommodation parameter is small enough in terms of the Knudsen number. Our proof uses the relative entropy method following closely [L. Saint-Raymond, Arch. Ration. Mech. Anal., 166, 47-80, 2003] in the case of the 3-torus, except for the boundary terms, which require special treatment.
\end{abstract}

Key words. Navier-Stokes Equations, Euler Equations, Boltzmann Equation, fluid dynamic limit, inviscid limit, slip coefficient, Maxwell's accommodation boundary condition, accommodation parameter, relative entropy method, dissipative solutions of the Euler Equations.

MSC 2000 subject classification. 35Q30, 82B40, 76D05, 76B99.

\section{Introduction}

In a program initiated more than 20 years ago with Dave Levermore $[3,4,5]$, we outlined a strategy for deriving incompressible fluid dynamic equations from the theory of renormalized solutions of the Boltzmann Equation invented by R. DiPerna and P.-L. Lions [12].

At the time of this writing, complete derivations of the Stokes [24, 25], StokesFourier [14], and Navier-Stokes-Fourier [15, 16, 20] Equations have been obtained, following that program, in the greatest possible generality allowed by the current existence theories for both the fluid dynamic and the Boltzmann Equations; see [36] for a survey of these issues.

The case of the incompressible Euler Equations in space dimension 3 stands out, primarily because there does not exist a satisfactory theory of global weak solutions of these equations analogous to Leray's theory of weak solutions of the Navier-Stokes Equations [21] in space dimension 3. Even if there was a global existence theory of weak solutions of the incompressible Euler Equations in the energy space $L_{t}^{\infty}\left(L_{x}^{2}\right)$ in dimension 3, such solutions would not satisfy the weak-strong uniqueness property

\footnotetext{
*Received: January 23, 2011; accepted: May 21, 2011.

†Université Paris-Diderot, Laboratoire J.-L. Lions, BP 187, 75252 Paris Cedex 05, France (claude.bardos@gmail.com).

$\ddagger$ Ecole Polytechnique, Centre de Mathématiques L. Schwartz, 91128 Palaiseau Cedex, France (golse@math.polytechnique.fr).

$\S$ Ecole Polytechnique, Centre de Mathématiques L. Schwartz, 91128 Palaiseau Cedex, France (lionel.paillard@club-internet.fr).
} 
observed by Leray in the case of the Navier-Stokes Equations. (Indeed there exist nontrivial compactly supported solutions of the incompressible Euler Equations in energy space; see [32, 33, 11].) In [23], P.-L. Lions proposed a notion of dissipative solution of the incompressible Euler Equations - in the same spirit of his definition of the notion of viscosity solutions of Hamilton-Jacobi Equations, but using the conservation of energy instead of the maximum principle as in the Hamilton-Jacobi case. The weak-strong uniqueness property is satisfied by dissipative solutions of the incompressible Euler Equations (essentially by definition): if there exists a classical $\left(C^{1}\right)$ solution of the incompressible Euler Equations, all dissipative solutions with the same initial data must coincide with this classical solution on its maximal time interval of existence. Unfortunately, dissipative solutions are not known to satisfy the incompressible Euler Equations in the sense of distributions.

Using the relative entropy method pioneered in [38] and adapted to the case of the Boltzmann Equation in [10, 25], L. Saint-Raymond [28, 29] succeeded in deriving dissipative solutions of the Euler Equations in arbitrary space dimension (or classical solutions whenever they exist) from weak solutions of the BGK model [28] or from renormalized solutions of the Boltzmann Equation [29].

However, all the derivations of fluid dynamic equations from the Boltzmann Equation referred to above are carried out in either the Euclidean space $\mathbf{R}^{N}$ or the flat torus $\mathbf{T}^{N}$ so as to avoid difficulties that may result from boundary conditions. The theory of renormalized solutions of the Boltzmann Equation in the presence of accommodation boundary conditions was obtained, only very recently, by S. Mischler [27]; subsequently, N. Masmoudi and L. Saint-Raymond established the Stokes-Fourier limit of such solutions [26].

In the present paper, we derive dissipative solutions (or classical solutions whenever they exist) of the incompressible Euler Equations from renormalized solutions of the Boltzmann Equation in some spatial domain satisfying Maxwell's accommodation boundary condition. In particular, we identify a sufficient scaling condition on the accommodation parameter under which the hydrodynamic limit of the family of solutions of the Boltzmann Equation is governed by the incompressible Euler Equations with its classical boundary condition - i.e. assuming that the velocity field is tangent at the boundary.

The outline of the paper is as follows. Section 2 gives a sufficient condition on the slip coefficient at the boundary under which the incompressible Euler Equations are obtained as the inviscid limit of the incompressible Navier-Stokes Equations with slip-boundary condition. The main result in this section is Theorem 2.2, and is based on an energy method. Section 3 provides a formal derivation of the incompressible Navier-Stokes Equations with slip boundary condition from the Boltzmann Equation with Maxwell's accommodation condition at the boundary of the spatial domain; see Theorem 3.1 for a precise statement of this result. Based on the intuition provided by sections 2 and 3, we identify a scaling limit of the Boltzmann Equation with Maxwell's accommodation boundary condition leading to the incompressible Euler Equations; see Theorem 4.2, whose proof occupies most of Section 4 .

It is a our great pleasure to offer this modest contribution to our friend Dave Levermore, in recognition of his outstanding influence on the analysis of nonlinear partial differential equations in the past 30 years, especially on the problem of hydrodynamic limits of the Boltzmann Equation, directly inspired from Hilbert's 6th problem on the axiomatization of physics. 
2. Inviscid limit of the Navier-Stokes Equations with slip boundary conditions

A a warm-up, we begin with a simple observation bearing on the inviscid limit of the incompressible Navier-Stokes Equations set in some smooth domain with slip boundary condition. In particular, we identify a sufficient scaling condition on the slip coefficient in order to obtain the incompressible Euler Equations in the inviscid limit.

Let $\Omega$ designate an open set in $\mathbf{R}^{N}$ with $C^{1}$ boundary $\partial \Omega$, assuming that $N=2$ or 3 ; henceforth the outward unit normal vector at the point $x$ of $\partial \Omega$ is denoted by $n_{x}$. Consider the following initial-boundary value problem with unknown $u_{\nu}=u_{\nu}(t, x)$, set for $x \in \Omega$ and $t \geq 0$ :

$$
\left\{\begin{array}{l}
\operatorname{div}_{x} u_{\nu}=0, \\
\partial_{t} u_{\nu}+\operatorname{div}_{x}\left(u_{\nu} \otimes u_{\nu}\right)+\nabla_{x} p_{\nu}=\nu \Delta_{x} u_{\nu}, \\
\left.u_{\nu} \cdot n\right|_{\partial \Omega}=0, \\
\left.\left(\nu\left(\Sigma\left(u_{\nu}\right) \cdot n\right)_{\tau}+\lambda u_{\nu}\right)\right|_{\partial \Omega}=0, \\
\left.u_{\nu}\right|_{t=0}=u^{i n},
\end{array}\right.
$$

where $\nu>0$ is the kinematic viscosity, $\lambda>0$ the slip coefficient,

$$
\Sigma(u):=\nabla_{x} u+\left(\nabla_{x} u\right)^{T},
$$

while

$$
v(x)_{\tau}:=\left(I-n(x)^{\otimes 2}\right) \cdot v(x) .
$$

Henceforth, we denote

$$
\mathcal{H}(\Omega):=\left\{v \in L^{2}\left(\Omega ; \mathbf{R}^{N}\right) \mid \operatorname{div} v=0 \text { and }\left.v \cdot n\right|_{\partial \Omega}=0\right\} .
$$

For each $\nu>0$ and $u^{i n} \in \mathcal{H}(\Omega)$, there exists a weak solution $u_{\nu}$ of (2.1) in $L^{\infty}\left(\mathbf{R}_{+} ; \mathcal{H}(\Omega)\right) \cap L^{2}\left(\mathbf{R}_{+} ; H^{1}(\Omega)\right)$, meaning that, for each test vector field $U$ belonging to $C\left(\mathbf{R}_{+} ; \mathcal{H}(\Omega)\right) \cap C_{c}^{\infty}\left(\mathbf{R}_{+} \times \bar{\Omega}\right)$, one has

$$
\begin{aligned}
& \nu \int_{0}^{\infty} \int_{\Omega} \frac{1}{2} \Sigma\left(u_{\nu}\right): \Sigma(U) d x d t+\lambda \int_{0}^{\infty} \int_{\partial \Omega} u_{\nu} \cdot U d S(x) d t \\
= & \int_{0}^{\infty} \int_{\Omega}\left(u_{\nu} \cdot \partial_{t} U+u_{\nu} \otimes u_{\nu}: \nabla_{x} U\right) d x d t+\int_{\Omega} u^{i n}(x) \cdot U(0, x) d x .
\end{aligned}
$$

This solution also satisfies $u_{\nu} \in C\left(\mathbf{R}_{+} ; w-L^{2}(\Omega)\right)$, together with the Leray-type energy dissipation inequality

$$
\begin{aligned}
\int_{\Omega} \frac{1}{2}\left|u_{\nu}(t, x)\right|^{2} d x & +\nu \int_{0}^{t} \int_{\Omega}\left|\Sigma\left(u_{\nu}\right)(t, x)\right|^{2} d x d t \\
& +\lambda \int_{0}^{t} \int_{\partial \Omega}\left|u_{\nu}(t, x)\right|^{2} d S(x) d t \leq \int_{\Omega} \frac{1}{2}\left|u^{i n}(x)\right|^{2} d x
\end{aligned}
$$

for each $t \geq 0$. Such a weak solution of (2.1) will henceforth be referred to as a "Leray solution of (2.1)". The well known classical theory of Leray solutions, in the case where the velocity field satisfies the Dirichlet boundary condition on $\partial \Omega$, can be adapted to the case of the slip-boundary condition; see [34, 7] and Theorem 2 in [18]. 
Since $u_{\nu} \in C\left(\mathbf{R}_{+} ; w-L^{2}(\Omega)\right)$, by an elementary density argument one can choose a sequence of test vector fields $U_{n}$ of the special form $U_{n}(t, x)=\chi_{n}(T-t) w(t, x)$, with $w \in C\left(\mathbf{R}_{+} ; \mathcal{H}(\Omega)\right) \cap C_{c}^{1}\left(\mathbf{R}_{+} \times \bar{\Omega}\right)$ and

$$
\chi_{n}(z)=\int_{-\infty}^{z} \chi_{n}^{\prime}(s) d s,
$$

where $\chi_{n}^{\prime}$ is a regularizing sequence on $\mathbf{R}$, so that the weak formulation (2.2) of the Navier-Stokes Equations becomes

$$
\begin{aligned}
& \nu \int_{0}^{T} \int_{\Omega} \frac{1}{2} \Sigma\left(u_{\nu}\right): \Sigma(w) d x d t+\lambda \int_{0}^{T} \int_{\partial \Omega} u_{\nu} \cdot w d S(x) d t \\
= & \int_{\Omega} u^{i n}(x) \cdot w(0, x) d x-\int_{\Omega} u_{\nu}(T, x) w(T, x) d x \\
& +\int_{0}^{T} \int_{\Omega}\left(u_{\nu} \cdot \partial_{t} w+u_{\nu} \otimes u_{\nu}: \nabla_{x} w\right) d x d t,
\end{aligned}
$$

for each $T>0$. Furthermore, denoting

$$
E(w):=\partial_{t} w+w \cdot \nabla_{x} w
$$

one has

while

$$
\int_{0}^{T} \int_{\Omega} u_{\nu} \cdot \partial_{t} w d x d t=\int_{0}^{T} \int_{\Omega}\left(u_{\nu} \cdot E(w)-u_{\nu} \otimes w \cdot \nabla_{x} w\right) d x d t
$$

$$
\begin{aligned}
& \int_{0}^{T} \frac{1}{2}|w(t, x)|^{2} d x-\int_{0}^{T} \frac{1}{2}|w(0, x)|^{2} d x+\int_{0}^{T} \int_{\Omega} w \otimes w: \nabla_{x} w d x d t \\
= & \int_{0}^{T} \int_{\Omega} w \cdot E(w) d x d t .
\end{aligned}
$$

Therefore

$$
\begin{aligned}
& \nu \int_{0}^{T} \int_{\Omega} \frac{1}{2} \Sigma\left(u_{\nu}\right): \Sigma(w) d x d t+\lambda \int_{0}^{T} \int_{\partial \Omega} u_{\nu} \cdot w d S(x) d t \\
= & \int_{0}^{T} \int_{\Omega}\left(u_{\nu} \cdot E(w)+u_{\nu} \otimes\left(u_{\nu}-w\right): \nabla_{x} w-w \otimes u_{\nu}: \nabla_{x} w\right) d x d t \\
& +\int_{\Omega} u^{i n}(x) \cdot w(0, x) d x-\int_{\Omega} u_{\nu}(T, x) w(T, x) d x,
\end{aligned}
$$

since

$$
\int_{\Omega} w \otimes u_{\nu}: \nabla_{x} w d x=\int_{\Omega} \operatorname{div}_{x}\left(u_{\nu} \frac{1}{2}|w|^{2}\right) d x=0
$$

because $\left.u_{\nu} \cdot n\right|_{\partial \Omega}=0$. Combining (2.5) and (2.4), we find that

$$
\begin{aligned}
& \nu \int_{0}^{T} \int_{\Omega} \frac{1}{2} \Sigma\left(u_{\nu}\right): \Sigma(w) d x d t+\lambda \int_{0}^{T} \int_{\partial \Omega} u_{\nu} \cdot w d S(x) d t \\
= & \int_{0}^{T} \int_{\Omega}\left(\left(u_{\nu}-w\right) \cdot E(w)+\left(u_{\nu}-w\right) \otimes\left(u_{\nu}-w\right): \nabla_{x} w\right) d x d t \\
& +\int_{\Omega} u^{i n}(x) \cdot w(0, x) d x-\int_{\Omega} \frac{1}{2}|w(0, x)|^{2} d x \\
& -\int_{\Omega} u_{\nu}(T, x) w(T, x) d x+\int_{\Omega} \frac{1}{2}|w(T, x)|^{2} d x .
\end{aligned}
$$


Finally, combining (2.3) and (2.6), we conclude that any Leray solution $u_{\nu}$ of $(2.1)$ satisfies the inequality

$$
\begin{aligned}
& \int_{\Omega} \frac{1}{2}\left|u_{\nu}-w\right|^{2}(t, x) d x+\int_{0}^{t} \int_{\Omega}\left(u_{\nu}-w\right) \otimes\left(u_{\nu}-w\right): \nabla_{x} w d x d s \\
& +\nu \int_{0}^{t} \int_{\Omega} \frac{1}{2}\left|\Sigma\left(u_{\nu}\right)(s, x)\right|^{2} d x d s+\lambda \int_{0}^{t} \int_{\partial \Omega}\left|u_{\nu}(s, x)\right|^{2} d S(x) d s \\
\leq & \int_{\Omega} \frac{1}{2}\left|u^{i n}(x)-w(0, x)\right|^{2} d x-\int_{0}^{t} \int_{\Omega} E(w) \cdot\left(u_{\nu}-w\right) d x d s \\
& +\nu \int_{0}^{t} \int_{\Omega} \frac{1}{2} \Sigma\left(u_{\nu}\right): \Sigma(w) d x d s+\lambda \int_{0}^{t} \int_{\partial \Omega} u_{\nu} \cdot w d S(x) d s
\end{aligned}
$$

for each $w \in C\left(\mathbf{R}_{+} ; \mathcal{H}(\Omega)\right) \cap C_{c}^{1}\left(\mathbf{R}_{+} \times \bar{\Omega}\right)$.

At this point we recall the definition of dissipative solutions of the incompressible Euler Equations set in a domain $\Omega$ with smooth boundary:

$$
\left\{\begin{array}{l}
\operatorname{div}_{x} u=0, \\
\partial_{t} u+\operatorname{div}_{x}(u \otimes u)+\nabla_{x} p=0, \\
\left.u \cdot n\right|_{\partial \Omega}=0 \\
\left.u\right|_{t=0}=u^{i n} .
\end{array}\right.
$$

Definition 2.1 (P.-L. Lions [23], p. 154, C. Bardos, E. Titi [6], p. 16). Given $u^{i n} \in \mathcal{H}(\Omega)$, a dissipative solution of (2.7) is an element $u \in C\left(\mathbf{R}_{+} ; w-\mathcal{H}(\Omega)\right)$ satisfying $\left.u\right|_{t=0}=u^{i n}$ and the inequality

$$
\begin{aligned}
& \int_{\Omega} \frac{1}{2}|u-w|^{2}(t, x) d x \\
\leq & \exp \left(\int_{0}^{t} 2\left\|\sigma(w)^{-}\right\|_{L^{\infty}(\Omega)}(s) d s\right) \int_{\Omega} \frac{1}{2}\left|u^{i n}(x)-w(0, x)\right|^{2} d x \\
& -\int_{0}^{t} \exp \left(\int_{s}^{t} 2\left\|\sigma(w)^{-}\right\|_{L^{\infty}(\Omega)}(\tau) d \tau\right) \int_{\Omega} E(w) \cdot(u-w)(s, x) d x d s
\end{aligned}
$$

for each $w \in C\left(\mathbf{R}_{+} ; \mathcal{H}(\Omega)\right) \cap C^{1}\left(\mathbf{R}_{+} \times \bar{\Omega}\right)$, where

$$
\sigma(w)^{-}(t, x):=\sup _{|\xi|=1}(-\Sigma(w)(t, x): \xi \otimes \xi) .
$$

We recall that, if the Euler Equations (2.7) have a classical solution $v$ belonging to $C^{1}\left(\left[0, T^{*}\right) \times \bar{\Omega}\right)$ and satisfying

$$
\sigma(v)^{-} \in L^{1}\left([0, T] ; L^{\infty}(\Omega)\right) \text { and } p \in L^{1}\left([0, T] ; H^{1}(\Omega)\right) \text { for each } T<T^{*},
$$

then all dissipative solutions of (2.7) must coincide with $v$ on $\left[0, T^{*}\right) \times \Omega$ a.e. since one can use $w=v$ as the test vector field, so that

$$
\int_{\Omega} E(v) \cdot(u-v)(s, x) d x=-\int_{\Omega} \nabla_{x} p \cdot(u-v)(s, x) d x=0
$$

because $(u-v)(s, \cdot) \in \mathcal{H}(\Omega)$ for each $s \in[0, T)$. 
THEOREM 2.2. Let $u^{i n} \in \mathcal{H}(\Omega)$, and assume that the slip coefficient $\lambda \equiv \lambda(\nu)$ in (2.1) scales with the kinematic viscosity $\nu$ so that

$$
\lambda(\nu) \rightarrow 0 \text { as } \nu \rightarrow 0 .
$$

Then any family $\left(u_{\nu}\right)$ of Leray solutions of (2.1) is relatively compact in $L^{\infty}\left(\mathbf{R}_{+} ; \mathcal{H}(\Omega)\right)$ weak- ${ }^{*}$ and in $C\left(\mathbf{R}_{+} ; w-\mathcal{H}(\Omega)\right)$ for the topology of uniform convergence on bounded time intervals, and each limit point of $\left(u_{\nu}\right)$ as $\nu \rightarrow 0$ is a dissipative solution of (2.7).

Proof. We deduce from (2.7) with $w=0$, or equivalently from the Leray energy inequality, that

$$
\begin{aligned}
& \sqrt{\nu} \Sigma\left(u_{\nu}\right) \text { is bounded in } L^{2}\left(\mathbf{R}_{+} ; L^{2}(\Omega)\right) \text {, and } \\
& \left.\sqrt{\lambda(\nu)} u_{\nu}\right|_{\partial \Omega} \text { is bounded in } L^{2}\left(\mathbf{R}_{+} ; L^{2}(\partial \Omega)\right) .
\end{aligned}
$$

By Gronwall's inequality

$$
\begin{aligned}
& \int_{\Omega} \frac{1}{2}\left|u_{\nu}-w\right|^{2}(t, x) d x \\
\leq & \exp \left(\int_{0}^{t} 2\left\|\sigma(w)^{-}\right\|_{L^{\infty}(\Omega)}(s) d s\right) \int_{\Omega} \frac{1}{2}\left|u^{i n}(x)-w(0, x)\right|^{2} d x \\
& -\int_{0}^{t} \exp \left(\int_{s}^{t} 2\left\|\sigma(w)^{-}\right\|_{L^{\infty}(\Omega)}(\tau) d \tau\right) \int_{\Omega} E(w) \cdot\left(u_{\nu}-w\right)(s, x) d x d s \\
& +\int_{0}^{t} \exp \left(\int_{s}^{t} 2\left\|\sigma(w)^{-}\right\|_{L^{\infty}(\Omega)}(\tau) d \tau\right) Q_{\nu}(s) d s
\end{aligned}
$$

where, by the Cauchy-Schwarz inequality,

$$
\begin{aligned}
Q_{\nu}(s)= & \nu\left\|\Sigma\left(u_{\nu}\right)\right\|_{L^{2}(\Omega)}(s)\|\Sigma(w)\|_{L^{2}(\Omega)}(s) \\
& +\lambda(\nu)\left\|u_{\nu}\right\|_{L^{2}(\partial \Omega)}(s)\|w\|_{L^{2}(\Omega)}(s) .
\end{aligned}
$$

In view of $(2.10)$, one has

$$
\left\|Q_{\nu}\right\|_{L^{1}([0, T])}=O(\sqrt{\nu})+O(\sqrt{\lambda(\nu)}) \rightarrow 0
$$

as $\nu \rightarrow 0$, and we conclude by passing to the limit in (2.11) following the same argument as in $[23]$.

Several remarks are in order after this result.

In some references, the slip boundary condition is written

$$
\left.\left(\nu\left(\frac{\partial u_{\nu}}{\partial n}\right)_{\tau}+\lambda u\right)\right|_{\partial \Omega}=0
$$

instead of

$$
\left.\left(\nu\left(\Sigma\left(u_{\nu}\right) \cdot n\right)_{\tau}+\lambda u\right)\right|_{\partial \Omega}=0 .
$$

Likewise, the boundary condition

$$
\operatorname{curl} u_{\nu} \times\left. n\right|_{\partial \Omega}=0
$$


is also considered by some authors - and referred to as the Navier slip condition in the context of the inviscid limit of the Navier-Stokes Equations; see for instance $[22,2,7,9]$.

If $\partial \Omega$ is a straight line, or a plane, or a hyperplane in space dimension $N>3$, the normal vector field $n$ is constant, so that

$$
\left.\left(\Sigma\left(u_{\nu}\right) \cdot n\right)_{\tau}\right|_{\partial \Omega}=\left.\left(\frac{\partial u_{\nu}}{\partial n}\right)_{\tau}\right|_{\partial \Omega}+\nabla^{\tau}\left(\left.u \cdot n\right|_{\partial \Omega}\right)=\left.\left(\frac{\partial u_{\nu}}{\partial n}\right)_{\tau}\right|_{\partial \Omega}
$$

and, in space dimension 3 ,

$$
\begin{aligned}
\operatorname{curl} u_{\nu} \times\left. n\right|_{\partial \Omega} & =\left.\left(\nabla_{x} u_{\nu}-\left(\nabla_{x} u\right)^{T}\right) \cdot n\right|_{\partial \Omega} \\
& =\left.\left(\frac{\partial u_{\nu}}{\partial n}\right)_{\tau}\right|_{\partial \Omega}-\nabla^{\tau}\left(\left.u \cdot n\right|_{\partial \Omega}\right)=\left.\left(\frac{\partial u_{\nu}}{\partial n}\right)_{\tau}\right|_{\partial \Omega}
\end{aligned}
$$

(with $\nabla^{\tau}$ denoting the tangential component of the $\nabla$ operator), since the velocity field $u_{\nu}$ is tangential on $\partial \Omega$. Therefore, in the case of a flat boundary, all these boundary conditions are equivalent.

If $\partial \Omega$ is a smooth curve, or a surface or a hypersurface in space dimension $N>3$, then

$$
\left.\left(\Sigma\left(u_{\nu}\right) \cdot n\right)_{\tau}\right|_{\partial \Omega}=\left.\left(\left(\frac{\partial u_{\nu}}{\partial n}\right)_{\tau}-\nabla^{\tau} n \cdot u_{\tau}\right)\right|_{\partial \Omega}
$$

while, in space dimension 3 ,

$$
\operatorname{curl} u_{\nu} \times\left. n\right|_{\partial \Omega}=\left.\left(\left(\frac{\partial u_{\nu}}{\partial n}\right)_{\tau}+\nabla^{\tau} n \cdot u_{\tau}\right)\right|_{\partial \Omega},
$$

so that all these boundary conditions differ by a 0 -order operator given by the Weingarten endomorphism of the boundary $\partial \Omega$.

Here we have chosen the second boundary condition above, as it is the more natural one when looking at the Navier-Stokes Equation as a fluid dynamic limit of the kinetic theory of gases.

However, the same argument as in the proof of Theorem 2.2 can be extended to treat the case of a slip coefficient $\lambda$ which is negative, provided that

$$
\lambda(\nu)_{+}=\max (\lambda(\nu), 0) \rightarrow 0 \quad \text { and } \quad \lambda_{-}(\nu)=\max (-\lambda(\nu), 0)=O(\nu)
$$

as $\nu \rightarrow 0$. Indeed, the contribution of $\lambda(\nu)_{-}$in the estimate (2.7) can be absorbed in the viscous dissipation term by means of the following classical inequality: for each $\alpha>0$, there exists $C_{\alpha}>0$ such that, for each $v \in H^{1}(\Omega)$,

$$
\int_{\partial \Omega}|v(x)|^{2} d S(x) \leq \alpha \int_{\Omega}\left|\nabla_{x} v(x)\right|^{2} d x+\frac{C_{\alpha}}{\alpha} \int_{\Omega}|v(x)|^{2} d x .
$$

With this observation, the term

$$
\left.\left(\Sigma\left(u_{\nu}\right) \cdot n\right)_{\tau}\right|_{\partial \Omega}
$$


can be replaced indifferently with either

$$
\left.\left(\left(\frac{\partial u_{\nu}}{\partial n}\right)_{\tau}\right)\right|_{\partial \Omega} \quad \text { or } \quad \operatorname{curl} u_{\nu} \times\left. n\right|_{\partial \Omega}
$$

in the slip boundary condition.

More precise variants of Theorem 2.2 have been established by various authors, see for instance $[37,8]$. The result given here holds for a very general class of nonnegative slip boundary coefficients $\lambda$ and is based upon the simplest imaginable energy estimate. The condition $\lambda \geq 0$ in (2.1) is somehow natural when this initial-boundary value problem is considered as some scaling limit of the Boltzmann Equation of the kinetic theory of gases.

Another question is whether the condition $\lambda(\nu) \rightarrow 0$ as $\nu \rightarrow 0$ is optimal. Consider instead the Dirichlet boundary condition for $u_{\nu}$, i.e.

$$
\left.u_{\nu}\right|_{\partial \Omega}=0 \text {. }
$$

Formally, this boundary condition corresponds with any one of the slip boundary conditions above with

$$
\varliminf_{\nu \rightarrow 0} \lambda(\nu)>0 .
$$

In that case, it well known that the Euler Equations (2.7) may fail to describe the inviscid limit of the Navier-Stokes Equations, even in the simpler 2 dimensional case. Because the Dirichlet boundary condition overdetermines the velocity field in the inviscid limit, the Euler Equations (2.7) are expected to govern the inviscid limit of the Navier-Stokes Equations only if the effect of viscosity remains confined on a thin layer near the boundary. But it may happen - and does happen under certain circumstances - that the viscous layer detaches from the boundary, as for instance in the case of the so-called von Karman vortex streets in the case of a NavierStokes flow past a cylinder, even at moderate Reynolds numbers. While this situation seems beyond the grasp of current mathematical analysis, there exists a least a very interesting criterion due to T. Kato [19], formulated in terms of the viscous energy dissipation only, identifying situations where the inviscid limit of the incompressible Navier-Stokes Equations with Dirichlet boundary condition is described by the Euler Equations. This suggests that, unless $\lambda(\nu) \rightarrow 0$, the Euler Equations (2.7) might also fail to govern the inviscid limit of the Navier-Stokes Equations with slip boundary conditions (2.1).

3. From the Boltzmann Equation with accommodation boundary condition to the Navier-Stokes Equations with slip boundary condition

In this section, we revisit the incompressible Navier-Stokes limit for the Boltzmann Equation in the case of the initial-boundary value problem. Our main interest is to understand how the slip boundary condition arises from Maxwell's accommodation boundary condition at the kinetic level in the fluid dynamic limit, and especially how the slip coefficient is related to the accommodation parameter. Strictly speaking, this is not needed in the proof of the main result in the present paper. Therefore, the discussion in this section will be only formal, along the line of [4].

Consider the Boltzmann Equation with the incompressible Navier-Stokes scaling

$$
\epsilon \partial_{t} F_{\epsilon}+v \cdot \nabla_{x} F_{\epsilon}=\frac{1}{\epsilon} \mathcal{B}\left(F_{\epsilon}, F_{\epsilon}\right) .
$$


Here the unknown is the distribution function $F \equiv F(t, x, v)$ that is the density at time $t$ of molecules with velocity $v \in \mathbf{R}^{N}$ at the position $x \in \Omega$ with respect to the phase space Lebesgue measure $d x d v$.

3.1. Formal structure of the Boltzmann Equation. The Boltzmann collision integral acts only on the $v$ variable in $F_{\epsilon}$, keeping $t, x$ as parameters. Its expression for $\phi \in C_{c}\left(\mathbf{R}^{N}\right)$ is

$$
\mathcal{B}(\phi, \phi)(v)=\iint_{\mathbf{R}^{N} \times \mathbf{S}^{N-1}}\left(\phi\left(v^{\prime}\right) \phi\left(v_{*}^{\prime}\right)-\phi(v) \phi\left(v_{*}\right)\right) b\left(v-v_{*}, \omega\right) d v_{*} d \omega,
$$

where $v^{\prime}, v_{*}^{\prime} \in \mathbf{R}^{N}$ are the velocities of 2 identical particles about to undergo an elastic collision, assuming that their post-collision velocities are $v, v_{*} \in \mathbf{R}^{N}$. The set of all possible pre-collision velocities $v^{\prime}, v_{*}^{\prime}$ are parametrized by the unit vector $\omega$ as follows:

$$
\left\{\begin{aligned}
& v^{\prime} \equiv v^{\prime}\left(v, v_{*}, \omega\right):=v-\left(v-v_{*}\right) \cdot \omega \omega, \\
& v_{*}^{\prime} \equiv v_{*}^{\prime}\left(v, v_{*}, \omega\right):=v_{*}+\left(v-v_{*}\right) \cdot \omega \omega .
\end{aligned}\right.
$$

The collision kernel $b(z, \omega)>0$ is a locally integrable function that satisfies the symmetries

$$
b\left(v-v_{*}, \omega\right)=b\left(v_{*}-v, \omega\right)=b\left(v^{\prime}-v_{*}^{\prime}, \omega\right)
$$

a.e. in $\left(v, v_{*}, \omega\right)$, assuming that $v^{\prime}$ and $v_{*}^{\prime}$ are given in terms of $v, v_{*}, \omega$ by the relations (3.3). Depending on the growth of the collision kernel $b$ as $\left|v-v_{*}\right| \rightarrow+\infty$, the collision integral can be extended by continuity to larger classes of functions than $C_{c}\left(\mathbf{R}^{N}\right)$. Finally, we denote

$$
\mathcal{B}(F, F)(t, x, v):=\mathcal{B}(F(t, x, \cdot), F(t, x, \cdot))(v) .
$$

The collision integral satisfies the identities

$$
\left\{\begin{aligned}
\int_{\mathbf{R}^{N}} \mathcal{B}(\phi, \phi)(v) d v & =0 \\
\int_{\mathbf{R}^{N}} \mathcal{B}(\phi, \phi)(v) v d v & =0 \\
\int_{\mathbf{R}^{N}} \mathcal{B}(\phi, \phi)(v)|v|^{2} d v & =0
\end{aligned}\right.
$$

for each $\phi \in C_{c}\left(\mathbf{R}^{N}\right)$ or in the larger class allowed by the growth at infinity of the collision kernel $b$. As a result, whenever $F$ is a classical solution of the scaled Boltzmann Equation (3.1) with appropriate decay as $|v| \rightarrow \infty$,

$$
\left\{\begin{array}{r}
\epsilon \partial_{t} \int_{\mathbf{R}^{N}} F_{\epsilon} d v+\operatorname{div}_{x} \int_{\mathbf{R}^{N}} v F_{\epsilon} d v=0 \\
\epsilon \partial_{t} \int_{\mathbf{R}^{N}} v F_{\epsilon} d v+\operatorname{div}_{x} \int_{\mathbf{R}^{N}} v^{\otimes 2} F_{\epsilon} d v=0 \\
\epsilon \partial_{t} \int_{\mathbf{R}^{N}} \frac{1}{2}|v|^{2} F_{\epsilon} d v+\operatorname{div}_{x} \int_{\mathbf{R}^{N}} v \frac{1}{2}|v|^{2} F_{\epsilon} d v=0
\end{array}\right.
$$

and these relations are the local conservation laws of mass, momentum, and energy respectively. 
Whenever $b\left(v-v_{*}, \omega\right)$ has polynomial growth as $\left|v-v_{*}\right| \rightarrow \infty$, for each positive, rapidly decaying $\phi \in C\left(\mathbf{R}^{N}\right)$ such that $\ln \phi$ has polynomial growth as $|v| \rightarrow \infty$, Boltzmann's $\mathrm{H}$ Theorem states that

$$
\int_{\mathbf{R}^{N}} \mathcal{B}(\phi, \phi)(v) \ln \phi(v) d v \leq 0
$$

and

$$
\begin{aligned}
\int_{\mathbf{R}^{N}} \mathcal{B}(\phi, \phi)(v) \ln \phi(v) d v=0 & \Leftrightarrow \mathcal{B}(\phi, \phi)=0 \\
& \Leftrightarrow \phi \text { is a Maxwellian distribution, }
\end{aligned}
$$

meaning that there exists $\rho, \theta>0$ and $u \in \mathbf{R}^{N}$ such that

$$
\phi(v)=\mathcal{M}_{\rho, u, \theta}(v):=\frac{\rho}{(2 \pi \theta)^{N / 2}} e^{-\frac{|v-u|^{2}}{2 \theta}}
$$

for all $v \in \mathbf{R}^{N}$. As a result, whenever $F$ is a classical solution of the scaled Boltzmann Equation (3.1) with appropriate decay as $|v| \rightarrow \infty$, it satisfies the differential entropy inequality

$$
\begin{aligned}
& \epsilon \partial_{t} \int_{\mathbf{R}^{N}} F_{\epsilon} \ln F_{\epsilon} d v+\operatorname{div}_{x} \int_{\mathbf{R}^{N}} v F_{\epsilon} \ln F_{\epsilon} d v \\
= & \frac{1}{\epsilon} \int_{\mathbf{R}^{N}} \mathcal{B}\left(F_{\epsilon}, F_{\epsilon}\right) \ln F_{\epsilon} d v \leq 0 .
\end{aligned}
$$

Throughout this paper, we denote

$$
M:=\mathcal{M}_{1,0,1}
$$

Since Maxwellians are equilibrium distributions for the collision integral, it is natural to investigate the linearization thereof about a Maxwellian, say $M$ for simplicity - the case of an arbitrary Maxwellian being similar. We therefore introduce the linearized collision operator, which has the form

$$
\mathcal{L}_{M} \phi:=-\left.M^{-1} \frac{\delta \mathcal{B}(F, F)}{\delta F}\right|_{F=M} \cdot M \phi
$$

i.e.

$$
\mathcal{L}_{M} \phi(v):=\iint_{\mathbf{R}^{N} \times \mathbf{S}^{N-1}}\left(\phi(v)+\phi\left(v_{*}\right)-\phi\left(v^{\prime}\right)-\phi\left(v_{*}^{\prime}\right)\right) b\left(v-v_{*}, \omega\right) M d v_{*} d \omega
$$

Likewise, we introduce the quadratic operator $\mathcal{Q}_{M}$ defined by

$$
\mathcal{Q}_{M}(\phi, \phi):=M^{-1} \mathcal{B}(M \phi, M \phi) \text {. }
$$

Under certain assumptions on the collision kernel $b$, known as Grad's angular cutoff assumption, H. Grad proved in [17] that $\mathcal{L}_{M}$ is an unbounded, self-adjoint Fredholm operator on $L^{2}\left(\mathbf{R}^{N} ; M d v\right)$ with domain

$$
\mathrm{D}\left(\mathcal{L}_{M}\right):=\left\{\phi \in L^{2}\left(\mathbf{R}^{N} ; M d v\right) \mid \phi\left(\bar{b} \star_{v} M\right) \in L^{2}\left(\mathbf{R}^{N} ; M d v\right)\right\},
$$


where

$$
\bar{b}(z):=\int_{\mathbf{S}^{N-1}} b(z, \omega) d \omega
$$

and $\star_{v}$ designates the convolution product in the $v$ variable. Moreover, the nullspace of $\mathcal{L}_{M}$ is

$$
\operatorname{Ker} \mathcal{L}_{M}=\operatorname{span}\left\{1, v_{1}, \ldots, v_{n},|v|^{2}\right\} .
$$

In particular, the tensor field $A(v)=v^{\otimes 2}-\frac{1}{N}|v|^{2}$ satisfies $A \perp \operatorname{Ker} \mathcal{L}_{M}$, so that, by the Fredholm alternative, there exists a unique tensor field

$$
\hat{A} \in \mathrm{D}\left(\mathcal{L}_{M}\right) \cap(\operatorname{Ker} \mathcal{L})^{\perp} \quad \text { such that } \mathcal{L}_{M} \hat{A}=A
$$

componentwise.

Henceforth in this paper, we assume that the collision kernel $b$ comes from a hard cutoff potential in the sense of Grad, and more precisely that it satisfies, for some constant $C_{b}>0$ and all $(z, \omega) \in \mathbf{R}^{N} \times \mathbf{S}^{N-1}$,

$$
0<b(z, \omega) \leq C_{b}(1+|z|), \quad \text { and } \bar{b}(z) \geq \frac{1}{C_{b}} .
$$

3.2. Boundary value problem and fluid dynamic limit. The incompressible Navier-Stokes limit of the Boltzmann Equation bears on solutions of the Boltzmann Equation that are of the form

$$
F_{\epsilon}=M\left(1+\epsilon g_{\epsilon}\right),
$$

where it is understood that the relative number density fluctuation $g_{\epsilon}$ is $O(1)$ in some sense to be made precise as $\epsilon \rightarrow 0$; see $[3,4,5]$ for more details, together with physical justifications for this scaling assumption.

Here, the scaled Boltzmann Equation (3.1) is set on the spatial domain $\Omega$, with Maxwell's accommodation at the boundary, that is assumed to be maintained at the constant temperature 1 . This boundary condition reads

$$
\begin{array}{r}
F_{\epsilon}(t, x, v)=(1-\alpha) \mathcal{R}_{x} F_{\epsilon}(t, x, v)+\alpha \Lambda_{x}\left(\frac{F_{\epsilon}}{M}\right)(t, x) M(v), \\
x \in \partial \Omega, v \cdot n_{x}<0,
\end{array}
$$

where

$$
\mathcal{R}_{x} F(t, x, v):=F\left(t, x, v-2 v \cdot n_{x} n_{x}\right)
$$

is the specular reflection operator on the boundary, while

$$
\Lambda_{x} \phi:=\sqrt{2 \pi} \int_{\mathbf{R}^{N}} \phi(v)\left(v \cdot n_{x}\right)_{+} M(v) d v .
$$

In (3.14), the parameter $\alpha$ satisfies $0 \leq \alpha \leq 1$, and is called the accommodation coefficient. The case $\alpha=0$ corresponds with specular reflection of the gas molecules on $\partial \Omega$ without thermal exchange, while the case $\alpha=1$ corresponds with diffuse reflection, or total accommodation, in which case gas molecules are instantaneously thermalized at the boundary. 
We henceforth denote, for each $\phi \in L^{1}\left(\mathbf{R}^{N} ; M d v\right)$,

$$
\langle\phi\rangle:=\int_{\mathbf{R}^{N}} \psi(v) M(v) d v .
$$

Theorem 3.1. Let $\left(F_{\epsilon}\right)_{\epsilon>0}$ be a family of solutions of the scaled Boltzmann Equation (3.1) set on the spatial domain $\Omega$, satisfying the accommodation boundary condition (3.14) on $\partial \Omega$. Assume that the relative fluctuations

$$
g_{\epsilon}=\frac{F_{\epsilon}-M}{\epsilon M} \rightarrow g
$$

a.e. and in weak- $L_{l o c}^{1}\left(\mathbf{R}_{+} \times \Omega \times \mathbf{R}^{N} ; d t d x M d v\right.$ ) (possibly up to extraction of a subsequence), and that

$$
\left\langle|v|^{3} \mathbf{1}_{|v|>R}\left|g_{\epsilon}\right|\right\rangle+\left\langle|\hat{A}| \mathbf{1}_{|v|>R}\left|\mathcal{Q}_{M}\left(g_{\epsilon}, g_{\epsilon}\right)\right|\right\rangle+\left\langle|\hat{A}||v| \mathbf{1}_{|v|>R}\left|g_{\epsilon}\right|\right\rangle \rightarrow 0
$$

in $L_{l o c}^{1}\left(\mathbf{R}_{+} \times \Omega\right)$ as $R \rightarrow+\infty$ uniformly in $\epsilon>0$. Then

$$
g(t, x, v)=\rho(t, x)+u(t, x) \cdot v+\theta(t, x) \frac{1}{2}\left(|v|^{2}-N\right),
$$

where $u$ is a solution of the incompressible Navier-Stokes Equations

$$
\left\{\begin{array}{l}
\operatorname{div}_{x} u=0, \\
\partial_{t} u+\operatorname{div}_{x}\left(u^{\otimes 2}\right)+\nabla_{x} p=\nu \Delta_{x} u
\end{array}\right.
$$

and where

$$
\nu=\frac{1}{(N-1)(N+2)}\langle\hat{A}: A\rangle
$$

Assume further that

$$
\left\{\begin{aligned}
\left\langle\left. v \cdot n_{x} g_{\epsilon}\right|_{\partial \Omega}\right\rangle & \rightarrow\left\langle\left. v \cdot n_{x} g\right|_{\partial \Omega}\right\rangle, \\
\left\langle\left. v_{\tau}\left(v \cdot n_{x}\right)_{+} g_{\epsilon}\right|_{\partial \Omega}\right\rangle & \rightarrow\left\langle\left. v_{\tau}\left(v \cdot n_{x}\right)_{+} g\right|_{\partial \Omega}\right\rangle
\end{aligned}\right.
$$

in weak- $L_{\text {loc }}^{1}\left(\mathbf{R}_{+} \times \partial \Omega\right)$. Then, the velocity field u satisfies the boundary condition

$$
\begin{cases}u \cdot n_{x}=0, & x \in \partial \Omega, \\ \nu\left(\Sigma(u) \cdot n_{x}\right)_{\tau}+\lambda u=0, & x \in \partial \Omega,\end{cases}
$$

where the slip coefficient is given by the formula

$$
\lambda=\frac{\alpha_{0}}{N-1}\left\langle\left|v_{\tau}\right|^{2}\left(v \cdot n_{x}\right)_{+}\right\rangle=\frac{\alpha_{0}}{\sqrt{2 \pi}} .
$$

Proof. Observe that, under the substitution $v \mapsto w=v-2 v \cdot n n$, one has, for each unit vector $n$,

$$
\int_{\mathbf{R}^{N}} \phi(v-2 v \cdot n n)(v \cdot n)_{-} M d v=\int_{\mathbf{R}^{N}} \phi(w)(w \cdot n)_{+} M d w,
$$

so that, for each $x \in \partial \Omega$, one has

$$
\begin{aligned}
\int_{\mathbf{R}^{N}} F_{\epsilon}(t, x, v) v \cdot n_{x} d v= & -\alpha \int_{\mathbf{R}^{N}} F_{\epsilon}(t, x, v)\left(v \cdot n_{x}\right)_{+} d v \\
& +\alpha \Lambda_{x}\left(\frac{F_{\epsilon}}{M}\right)(t, x) \int_{\mathbf{R}^{N}} M(v)\left(v \cdot n_{x}\right)_{-} d v=0,
\end{aligned}
$$


since

$$
\int_{\mathbf{R}^{N}} M(v)\left(v \cdot n_{x}\right)_{+} d v=\int_{\mathbf{R}^{N}} M(v)\left(v \cdot n_{x}\right)_{-} d v=\frac{1}{\sqrt{2 \pi}} .
$$

Hence, for each $x \in \partial \Omega$ and each $\epsilon>0$, one has

$$
\left\langle v g_{\epsilon}\right\rangle(t, x) \cdot n_{x}=\frac{1}{\epsilon} \int_{\mathbf{R}^{N}} F_{\epsilon}(t, x, v) v \cdot n_{x} d v=0,
$$

so that, after passing to the limit as $\epsilon \rightarrow 0$,

$$
u(t, x) \cdot n_{x}=\langle v g\rangle(t, x) \cdot n_{x}=0, \quad t>0, x \in \partial \Omega .
$$

Next write the local conservation of momentum - the second local conservation law in (3.6) - in the form

$$
\partial_{t}\left\langle v g_{\epsilon}\right\rangle+\operatorname{div}_{x} \frac{1}{\epsilon}\left\langle A g_{\epsilon}\right\rangle+\nabla_{x} \frac{1}{\epsilon}\left\langle\frac{1}{N}|v|^{2} g_{\epsilon}\right\rangle=0,
$$

where

$$
A \equiv A(v):=v^{\otimes 2}-\frac{1}{N}|v|^{2} .
$$

Let now $w \equiv w(x) \in \mathbf{R}^{N}$ designate a compactly supported $C^{1}$ vector field on $\mathbf{R}^{N}$ satisfying

$$
\operatorname{div} w=0 \quad \text { and } \quad w(x) \cdot n_{x}=0, x \in \partial \Omega .
$$

Taking the inner product of both sides of the local conservation of momentum with $w$ and integrating over $\Omega$ leads to

$$
\begin{aligned}
\partial_{t} \int_{\Omega} w \cdot\left\langle v g_{\epsilon}\right\rangle d x+\int_{\partial \Omega} w & \otimes n_{x}: \frac{1}{\epsilon}\left\langle A g_{\epsilon}\right\rangle d S(x) \\
& -\int_{\Omega} \nabla w: \frac{1}{\epsilon}\left\langle A g_{\epsilon}\right\rangle d x=0,
\end{aligned}
$$

since

$$
\begin{aligned}
\int_{\Omega} w \cdot \nabla_{x} \frac{1}{\epsilon}\left\langle\frac{1}{N}|v|^{2} g_{\epsilon}\right\rangle d x & =\int_{\Omega} \operatorname{div}_{x}\left(\frac{1}{\epsilon}\left\langle\frac{1}{N}|v|^{2} g_{\epsilon}\right\rangle \cdot w\right) d x \\
& =\int_{\partial \Omega} \frac{1}{\epsilon}\left\langle\frac{1}{N}|v|^{2} g_{\epsilon}\right\rangle w \cdot n_{x} d S(x)=0 .
\end{aligned}
$$

Next we pass to the limit in the sense of distributions in each term appearing in (3.17); following the analysis in $[3,4]$, one finds that

$$
\int_{\Omega} w \cdot\left\langle v g_{\epsilon}\right\rangle d x \rightarrow \int_{\Omega} w \cdot\langle v g\rangle d x=\int_{\Omega} w \cdot u d x
$$

while

$$
\begin{aligned}
& \int_{\Omega} \nabla w: \frac{1}{\epsilon}\left\langle A g_{\epsilon}\right\rangle d x \rightarrow \int_{\Omega} \nabla w:(A(u)-\nu \Sigma(u)) d x \\
= & \int_{\Omega} \nabla w:\left(u^{\otimes 2}-\nu \Sigma(u)\right) d x .
\end{aligned}
$$


(Indeed, since $w$ is divergence-free, $\nabla w: \frac{1}{N}|u|^{2}=\frac{1}{N}|u|^{2} \operatorname{div}_{x} w=0$.)

It remains to analyze the boundary term

$$
\int_{\partial \Omega} w \otimes n_{x}: \frac{1}{\epsilon}\left\langle A g_{\epsilon}\right\rangle d S(x) .
$$

Since $w \cdot n_{x}=0$ on $\partial \Omega$,

$$
w \otimes n_{x}:\left\langle A g_{\epsilon}\right\rangle=w \otimes n_{x}:\left\langle v^{\otimes 2} g_{\epsilon}\right\rangle=\left\langle v_{\tau} v \cdot n_{x} g_{\epsilon}\right\rangle \cdot w .
$$

At this point, we decompose the boundary term into the contribution of gas molecules about to collide and those having just collided with the boundary

$$
\left\langle v_{\tau} v \cdot n_{x} g_{\epsilon}\right\rangle=\left\langle v_{\tau} v \cdot n_{x} \mathbf{1}_{v \cdot n_{x}>0} g_{\epsilon}\right\rangle+\left\langle v_{\tau} v \cdot n_{x} \mathbf{1}_{v \cdot n_{x}<0} g_{\epsilon}\right\rangle
$$

and use the accommodation condition to write

$$
\left\langle v_{\tau} v \cdot n_{x} \mathbf{1}_{v \cdot n_{x}<0} g_{\epsilon}\right\rangle=\left\langle v_{\tau} v \cdot n_{x} \mathbf{1}_{v \cdot n_{x}<0}\left((1-\alpha) \mathcal{R}_{x} g_{\epsilon}+\alpha \Lambda_{x}\left(g_{\epsilon}\right)\right)\right\rangle .
$$

Observing that

$$
\begin{aligned}
\left\langle v_{\tau} v \cdot n_{x} \mathbf{1}_{v \cdot n_{x}<0} \mathcal{R}_{x} g_{\epsilon}\right\rangle & =\left\langle\mathcal{R}_{x}\left(v_{\tau} v \cdot n_{x} \mathbf{1}_{v \cdot n_{x}>0}\right) g_{\epsilon}\right\rangle \\
& =-\left\langle v_{\tau} v \cdot n_{x} \mathbf{1}_{v \cdot n_{x}>0} g_{\epsilon}\right\rangle
\end{aligned}
$$

we conclude that

$$
\left\langle v_{\tau} v \cdot n_{x} \mathbf{1}_{v \cdot n_{x}<0} g_{\epsilon}\right\rangle=-\left\langle v_{\tau} v \cdot n_{x} \mathbf{1}_{v \cdot n_{x}>0}\left((1-\alpha) g_{\epsilon}+\alpha \Lambda\left(g_{\epsilon}\right)\right)\right\rangle,
$$

so that

$$
\left\langle v_{\tau} v \cdot n_{x} g_{\epsilon}\right\rangle=\alpha\left\langle v_{\tau} v \cdot n_{x} \mathbf{1}_{v \cdot n_{x}>0}\left(g_{\epsilon}-\Lambda_{x}\left(g_{\epsilon}\right)\right)\right\rangle=\alpha\left\langle v_{\tau} v \cdot n_{x} \mathbf{1}_{v \cdot n_{x}>0} g_{\epsilon}\right\rangle,
$$

where the second equality follows from the fact that the function

$$
v \mapsto v_{\tau} v \cdot n_{x} \mathbf{1}_{v \cdot n_{x}>0}\left(g_{\epsilon}-\Lambda_{x}\left(g_{\epsilon}\right)\right.
$$

is odd in $v_{\tau}$.

Therefore the boundary term appearing in (3.17) becomes

$$
\int_{\partial \Omega} w \otimes n_{x}: \frac{1}{\epsilon}\left\langle A g_{\epsilon}\right\rangle d S(x)=\frac{\alpha}{\epsilon} \int_{\partial \Omega} w \cdot\left\langle v_{\tau} v \cdot n_{x} \mathbf{1}_{v \cdot n_{x}>0} g_{\epsilon}\right\rangle d S(x) .
$$

Assume that $\alpha \equiv \alpha(\epsilon)$ varies with $\epsilon$ so that $\alpha(\epsilon) / \epsilon \rightarrow \alpha_{0}$ as $\epsilon \rightarrow 0$. Since

$$
g_{\epsilon} \rightarrow g=\rho+u \cdot v+\theta \frac{1}{2}\left(|v|^{2}-N\right),
$$

and we already know that

$$
u \cdot n_{x}=0 \quad \text { on } \partial \Omega,
$$

one has

$$
\begin{aligned}
& w \cdot\left\langle v_{\tau} v \cdot n_{x} \mathbf{1}_{v \cdot n_{x}>0} g_{\epsilon}\right\rangle \rightarrow\left\langle v_{\tau}^{\otimes 2}\left(v \cdot n_{x}\right)_{+}\right\rangle: u_{t} \otimes w \\
= & \frac{1}{N-1}\left\langle\left|v_{\tau}\right|^{2}\left(v \cdot n_{x}\right)_{+}\right\rangle u \cdot w .
\end{aligned}
$$


Thus, passing to the limit in (3.17) leads to

$$
\begin{aligned}
\partial_{t} \int_{\Omega} w \cdot u d x+\frac{\alpha_{0}}{N-1}\left\langle\left|v_{\tau}\right|^{2}\left(v \cdot n_{x}\right)_{+}\right\rangle \int_{\partial \Omega} u \cdot w d S(x) \\
-\int_{\Omega} \nabla w:\left(u^{\otimes 2}-\nu \Sigma(u)\right) d x=0 .
\end{aligned}
$$

(Notice that the term $\left\langle\left|v_{\tau}\right|^{2}\left(v \cdot n_{x}\right)_{+}\right\rangle$is independent of $x$ and therefore comes out of the boundary integral.)

Whenever $u(t, \cdot) \in C^{2}(\bar{\Omega})$, an application of Green's formula transforms the last integral above into

$$
\begin{aligned}
\int_{\Omega} \nabla w:\left(u^{\otimes 2}-\nu \Sigma(u)\right) d x= & -\int_{\Omega} w \cdot\left(\operatorname{div}_{x}\left(u^{\otimes 2}\right)-\nu \operatorname{div}_{x}(\Sigma(u))\right) d x \\
& +\int_{\partial \Omega} w \cdot u u \cdot n_{x} d S(x)-\nu \int_{\partial \Omega} w \cdot(\Sigma(u) \cdot n) d S(x) .
\end{aligned}
$$

Since $u \cdot n_{x}=0$ on $\partial \Omega$, the second integral on the right-hand side above vanishes, and since $\operatorname{div}_{x} u=0$, one has $\operatorname{div}_{x}(\Sigma(u))=\Delta_{x} u$, so that

$$
\begin{aligned}
\int_{\Omega} \nabla w:\left(u^{\otimes 2}-\nu \Sigma(u)\right) d x= & -\int_{\Omega} w \cdot\left(\operatorname{div}_{x}\left(u^{\otimes 2}\right)-\nu \Delta_{x} u\right) d x \\
& -\nu \int_{\partial \Omega} w \cdot(\Sigma(u) \cdot n) d S(x) .
\end{aligned}
$$

Thus, if $u \in C^{2}([0, T] \times \bar{\Omega})$, the equality (3.18) becomes

$$
\begin{aligned}
& \partial_{t} \int_{\Omega} w \cdot u d x+\int_{\Omega} w \cdot\left(\operatorname{div}_{x}\left(u^{\otimes 2}\right)-\nu \Delta_{x} u\right) d x \\
& \quad+\frac{\alpha_{0}}{N-1}\left\langle\left|v_{\tau}\right|^{2}\left(v \cdot n_{x}\right)_{+}\right\rangle \int_{\partial \Omega} u \cdot w d S(x)+\nu \int_{\partial \Omega} w \cdot(\Sigma(u) \cdot n) d S(x)=0 .
\end{aligned}
$$

This identity holds, say, for each $w \in C_{c}^{\infty}\left(\bar{\Omega} ; \mathbf{R}^{N}\right)$. In particular, it holds for each $w \in C_{c}^{\infty}\left(\Omega ; \mathbf{R}^{N}\right)$ such that $\operatorname{div} w=0$, which implies that

$$
\partial_{t} u+\operatorname{div}_{x}\left(u^{\otimes 2}\right)-\nu \Delta_{x} u=-\nabla_{x} p
$$

in the sense of distributions (for some $p \in \mathcal{D}^{\prime}\left(\mathbf{R}_{+}^{*} \times \Omega\right)$ ). Since the velocity field $u$ belongs to $C^{2}([0, T] \times \bar{\Omega})$ we conclude that $p \in C^{1}([0, T] \times \bar{\Omega})$. Substituting this in the identity above with $w \in C_{c}^{\infty}\left(\bar{\Omega} ; \mathbf{R}^{N}\right)$, such that $\operatorname{div} w=0$ and $w \cdot n=0$ on $\partial \Omega$, gives

$$
-\int_{\Omega} w \cdot \nabla_{x} p d x+\int_{\partial \Omega}\left(\nu \Sigma(u) \cdot n_{x}+\frac{\alpha_{0}}{N-1}\left\langle\left|v_{\tau}\right|^{2}\left(v \cdot n_{x}\right)_{+}\right\rangle u\right) \cdot w d S(x)=0
$$

and since, by Green's formula,

$$
-\int_{\Omega} w \cdot \nabla_{x} p d x=-\int_{\Omega} \operatorname{div}_{x}(p w) d x=\int_{\partial \Omega} p w \cdot n_{x} d S(x)=0
$$

we conclude that

$$
\nu\left(\Sigma(u) \cdot n_{x}\right)_{\tau}+\frac{\alpha_{0}}{N-1}\left\langle\left|v_{\tau}\right|^{2}\left(v \cdot n_{x}\right)_{+}\right\rangle u=0 \quad \text { on } \partial \Omega .
$$


In other words, (3.18) is the weak formulation of

$$
\left\{\begin{array}{lll}
\partial_{t} u+\operatorname{div}_{x}\left(u^{\otimes 2}\right)-\nu \Delta_{x} u=-\nabla_{x} p, & x \in \Omega, & t>0, \\
\nu\left(\Sigma(u) \cdot n_{x}\right)_{\tau}+\lambda u=0, & x \in \partial \Omega, & t>0, \\
u \cdot n_{x}=0, & x \in \partial \Omega, & t>0,
\end{array}\right.
$$

with

$$
\lambda=\frac{\alpha_{0}}{N-1}\left\langle\left|v_{\tau}\right|^{2}\left(v \cdot n_{x}\right)_{+}\right\rangle=\frac{\alpha_{0}}{\sqrt{2 \pi}} .
$$

The argument above is a proof of the (formal) Navier-Stokes limit Theorem 3.1 by a moment method analogous to the one used in [4]. As far as we know, the first derivation of this slip boundary condition, in the steady, linearized regime (i.e. leading to the Stokes Equations in the fluid limit), is due to K. Aoki, T. Inamuro, and Y. Onishi [1] (see especially formula (33) in that reference). That derivation uses a Hilbert expansion method (formal series expansion of the solution of the Boltzmann Equation in powers of the Knudsen number $\epsilon$ ). The interested reader is referred to the recent book by Y. Sone [35] (in particular to $§ 3.7$ there) for a systematic study of boundary conditions in the context of the fluid dynamic limit of the Boltzmann Equation.

For a complete proof of the derivation of the same slip boundary condition as in Theorem 3.1 in the linearized regime - i.e. in a situation where the limiting equation is the Stokes, instead of the Navier-Stokes Equations - the reader is referred to the work of N. Masmoudi and L. Saint-Raymond [26].

4. From the Boltzmann Equation with accommodation boundary condition to the incompressible Euler Equations

In this section, we consider the Boltzmann Equation in the scaling leading to the incompressible Euler Equations in the fluid dynamic limit. We recall from $[3,4]$ that this scaling is

$$
\epsilon \partial_{t} F_{\epsilon}+v \cdot \nabla_{x} F_{\epsilon}=\frac{1}{\epsilon^{1+q}} \mathcal{B}\left(F_{\epsilon}, F_{\epsilon}\right), \quad(x, v) \in \Omega \times \mathbf{R}^{N}
$$

with $q>0$, while the distribution function $F_{\epsilon}$ is sought in the same form (3.13) as in the Navier-Stokes limit. This scaled Boltzmann Equation is supplemented with Maxwell's accommodation condition on $\partial \Omega$, with accommodation coefficient $\alpha \equiv \alpha(\epsilon)$ driven by the small parameter $\epsilon$ :

$$
\begin{array}{r}
F_{\epsilon}(t, x, v)=(1-\alpha(\epsilon)) \mathcal{R}_{x} F_{\epsilon}(t, x, v)+\alpha(\epsilon) \Lambda \\
\left(\frac{F_{\epsilon}}{M}\right)(t, x) M(v), \\
x \in \partial \Omega, v \cdot n_{x}<0,
\end{array}
$$

and with the initial condition

$$
F_{\epsilon}(0, x, v)=F_{\epsilon}^{i n}(x, v), \quad(x, v) \in \Omega \times \mathbf{R}^{N} .
$$

The formal result presented in Theorem 3.1 suggests that, in the limit as $\epsilon \rightarrow 0$, the velocity field

$$
\lim _{\epsilon \rightarrow 0} \frac{1}{\epsilon} \int_{\mathbf{R}^{N}} v F_{\epsilon} d v
$$


should behave like the solution of the incompressible Navier-Stokes Equations with kinematic viscosity of order $\epsilon^{q}$ and slip boundary condition with slip coefficient of the order of $\alpha(\epsilon) / \epsilon$. Thus, if $\alpha(\epsilon)=o(\epsilon)$, Theorem 2.2 suggests that this velocity field should satisfy the incompressible Euler Equation (2.7). In fact, the formal result in Theorem 3.1 is only a guide for our intuition, and we shall give a direct proof of the Euler limit starting from the Boltzmann Equation with accommodation boundary condition without using the Navier-Stokes limit.

4.1. Renormalized solutions and a priori estimates. Global solutions of the Cauchy problem for the Boltzmann Equation for initial data of arbitrary size have been constructed by R. DiPerna and P.-L. Lions [12]. Their theory of renormalized solutions was extended to the initial boundary value problem by S. Mischler [27]. His result is summarized below; see also Section 2 in [26] and Section 2.3.2 of [31].

TheOREM 4.1 (Mischler). Let $F_{\epsilon}^{i n} \equiv F_{\epsilon}^{i n}(x, v) \geq 0$ a.e. on $\Omega \times \mathbf{R}^{N}$ be a measurable function satisfying

$$
\iint_{\Omega \times \mathbf{R}^{N}}\left(1+|v|^{2}+\left|\ln F_{\epsilon}^{i n}(x, v)\right|\right) F_{\epsilon}^{i n}(x, v) d x d v<+\infty .
$$

There exists $F_{\epsilon} \in C\left(\mathbf{R}_{+} ; L^{1}\left(\Omega \times \mathbf{R}^{N}\right)\right)$ satisfying the initial condition (4.3), and the Boltzmann Equation (4.1) together with the boundary condition (4.2) in the renormalized sense, meaning that, for each $\Gamma \in C^{1}\left(\mathbf{R}_{+}\right)$such that $Z \mapsto \sqrt{1+Z} \Gamma^{\prime}(Z)$ is bounded on $\mathbf{R}_{+}$, the function

$$
\Gamma^{\prime}\left(\frac{F_{\epsilon}}{M}\right) \mathcal{B}\left(F_{\epsilon}, F_{\epsilon}\right) \in L_{l o c}^{1}\left(\mathbf{R}_{+} \times \bar{\Omega} \times \mathbf{R}^{N}\right)
$$

and

$$
\begin{aligned}
& \int_{0}^{\infty} \iint_{\Omega \times \mathbf{R}^{N}} \Gamma\left(\frac{F_{\epsilon}}{M}\right)\left(\epsilon \partial_{t}+v \cdot \nabla_{x}\right) \phi M d v d x d t \\
& +\frac{1}{\epsilon^{1+q}} \int_{0}^{\infty} \iint_{\Omega \times \mathbf{R}^{N}} \Gamma^{\prime}\left(\frac{F_{\epsilon}}{M}\right) \mathcal{B}\left(F_{\epsilon}, F_{\epsilon}\right) \phi d v d x d t \\
= & \int_{0}^{\infty} \iint_{\partial \Omega \times \mathbf{R}^{N}} \Gamma\left(\frac{F_{\epsilon}}{M}\right) \phi v \cdot n_{x} M d v d S(x) d t \\
& -\left.\epsilon \iint_{\Omega \times \mathbf{R}^{N}} \Gamma\left(\frac{F_{\epsilon}^{i n}}{M}\right) \phi\right|_{t=0} M d v d x
\end{aligned}
$$

for each $\phi \in C_{c}^{1}\left(\mathbf{R}_{+} \times \bar{\Omega} \times \mathbf{R}^{N}\right)$.

Moreover

a) the trace of $F_{\epsilon}$ on $\partial \Omega$ satisfies the accommodation boundary condition

$$
\left.F_{\epsilon}\right|_{\partial \Omega}(t, x, v)=(1-\alpha) \mathcal{R}_{x}\left(\left.F_{\epsilon}\right|_{\partial \Omega}\right)(t, x, v)+\Lambda_{x}\left(\frac{\left.F_{\epsilon}\right|_{\partial \Omega}}{M}\right)(t, x) M(v)
$$

for a.e. $(t, x, v) \in \mathbf{R}_{+} \times \partial \Omega \times \mathbf{R}^{N}$ such that $v \cdot n_{x}>0$;

b) the distribution function $F_{\epsilon}$ satisfies the local conservation law of mass

$$
\epsilon \partial_{t} \int_{\mathbf{R}^{N}} F_{\epsilon} d v+\operatorname{div}_{x} \int_{\mathbf{R}^{N}} v F_{\epsilon} d v=0
$$


with boundary condition

$$
\int_{\mathbf{R}^{N}} F_{\epsilon}(t, x, v) v \cdot n_{x} d v=0, \quad x \in \partial \Omega, t>0
$$

c) the distribution function $F_{\epsilon}$ satisfies the relative entropy inequality

$$
\begin{aligned}
& H\left(F_{\epsilon} \mid M\right)(t)-H\left(F_{\epsilon}^{i n} \mid M\right) \\
\leq & -\frac{1}{\epsilon^{2+q}} \int_{0}^{t} \int_{\Omega} \mathcal{P}_{\epsilon}(s, x) d x d s-\frac{\alpha}{\epsilon} \int_{0}^{t} \int_{\partial \Omega} \mathcal{D G}_{\epsilon}(s, x) d x d s
\end{aligned}
$$

for each $t>0$, where the following notations have been used: for each $f, g$ measurable on $\Omega \times \mathbf{R}^{N}$ such that $f \geq 0$ and $g>0$ a.e., the relative entropy is

$$
H(f \mid g):=\iint_{\Omega \times \mathbf{R}^{N}} h\left(\frac{f}{g}-1\right) g d v d x
$$

with

$$
h(z):=(1+z) \ln (1+z)-z,
$$

while the entropy production rate per unit volume is

$$
\mathcal{P}_{\epsilon}:=\iiint_{\mathbf{R}^{N} \times \mathbf{R}^{N} \times \mathbf{S}^{N-1}} r\left(\frac{F_{\epsilon}^{\prime} F_{\epsilon *}^{\prime}}{F_{\epsilon} F_{\epsilon *}}-1\right) F_{\epsilon} F_{\epsilon *} b\left(v-v_{*}, \omega\right) d v d v_{*} d \omega
$$

with

$$
r(z):=z \ln (1+z) \geq 0,
$$

and the Darrozes-Guiraud information is

$$
\mathcal{D} \mathcal{G}_{\epsilon}:=\frac{1}{\sqrt{2 \pi}}\left(\Lambda_{x}\left(h\left(\frac{F_{\epsilon}}{M}-1\right)\right)-h\left(\Lambda_{x}\left(\frac{F_{\epsilon}}{M}-1\right)\right)\right) .
$$

In particular $H(f \mid g) \geq 0$ since $h \geq 0$ on $[-1,+\infty)$ and $\mathcal{P}_{\epsilon} \geq 0$ a.e. on $\mathbf{R}_{+} \times \Omega$ since $r \geq 0$ on $(-1,+\infty)$, while $\mathcal{D} \mathcal{G}_{\epsilon} \geq 0$ a.e. on $\mathbf{R}_{+} \times \partial \Omega$ since $h$ is convex and $\Lambda_{x}$ is the average with respect to a probability measure;

d) for each $T>0$ and each compact $K \subset \partial \Omega$, there exists $C_{K, T}>0$ such that, for each $\epsilon>0$, one has

$$
\int_{0}^{T} \iint_{K \times \mathbf{R}^{N}} F_{\epsilon}(t, x, v)\left(v \cdot n_{x}\right)^{2} d v d S(x) d t \leq C_{K, T}, \quad \epsilon>0 .
$$

Statement d) appears in [26], without proof. We give a brief justification for this estimate below.

Notice that in general, renormalized solutions of the initial-boundary value problem (4.1)-(4.2)-(4.3) are not known to satisfy the local conservation law of momentum; see Equation (2.35) in [31] for a variant involving a defect measure, following an earlier remark due to P.-L. Lions and N. Masmoudi. 
On the contrary, for any classical solution $F_{\epsilon} \in C\left(\mathbf{R}_{+} \times \bar{\Omega} \times \mathbf{R}^{N}\right)$ that is continuously differentiable in $(t, x)$ and such that

$$
\begin{aligned}
& v \mapsto \sup _{\substack{0 \leq t \leq T \\
|x| \leq R}}\left(\left|F_{\epsilon}(t, x, v)\right|+\left|\partial_{t} F_{\epsilon}(t, x, v)\right|+\left|\nabla_{x} F_{\epsilon}(t, x, v)\right|\right) \\
& \text { decays rapidly as }|v| \rightarrow+\infty
\end{aligned}
$$

one has

$$
\epsilon \partial_{t} \int_{\mathbf{R}^{N}} v F_{\epsilon} d v+\operatorname{div}_{x} \int_{\mathbf{R}^{N}} v^{\otimes 2} F_{\epsilon} d v=0, \quad x \in \Omega, t>0 .
$$

Moreover, for each $w \in C_{c}^{1}\left(\mathbf{R}_{+} \times \bar{\Omega}\right)$, Green's formula implies that

$$
\begin{aligned}
& \int_{0}^{t} \iint_{\Omega \times \mathbf{R}^{N}}\left(\epsilon v \cdot \partial_{t} w(s, x)+v^{\otimes 2}: \nabla_{x} w(s, x)\right) F_{\epsilon}(s, x, v) d v d x d s \\
= & \int_{0}^{t} \int_{\partial \Omega \times \mathbf{R}^{N}} v^{\otimes 2}: w(s, x) \otimes n_{x} F_{\epsilon}(s, x, v) d v d S(x) d s \\
& +\epsilon \iint_{\Omega \times \mathbf{R}^{N}} w(t, x) \cdot v F_{\epsilon}(t, x, v) d v d x \\
& -\epsilon \iint_{\Omega \times \mathbf{R}^{N}} w(0, x) \cdot v F_{\epsilon}^{i n}(x, v) d v d x .
\end{aligned}
$$

Let us use the accommodation condition (4.2) to reduce the boundary integral:

$$
\begin{aligned}
& \int_{\mathbf{R}^{N}} v^{\otimes 2}: w(t, x) \otimes n_{x} F_{\epsilon}(s, x, v) d v \\
= & \int_{\mathbf{R}^{N}}(w(s, x) \cdot v)\left(v \cdot n_{x}\right)_{+} F_{\epsilon}(s, x, v) d v \\
& -\int_{\mathbf{R}^{N}}(w(s, x) \cdot v)\left(v \cdot n_{x}\right)_{-} F_{\epsilon}(s, x, v) d v
\end{aligned}
$$

and, whenever $w$ is tangential on $\partial \Omega$, one has

$$
\begin{aligned}
& \int_{\mathbf{R}^{N}}\left(w(s, x) \cdot n_{x}\right)_{-} F_{\epsilon}(s, x, v) d v \\
= & \int_{\mathbf{R}^{N}}(w(s, x) \cdot v)\left(v \cdot n_{x}\right)_{-}\left((1-\alpha) \mathcal{R}_{x} F_{\epsilon}+\alpha \Lambda_{x}\left(\frac{F_{\epsilon}}{M}\right) M\right)(s, x, v) d v \\
= & \int_{\mathbf{R}^{N}}(w(s, x) \cdot v)\left(v \cdot n_{x}\right)_{+}\left((1-\alpha) F_{\epsilon}+\alpha \Lambda_{x}\left(\frac{F_{\epsilon}}{M}\right) M\right)(s, x, v) d v .
\end{aligned}
$$

Therefore

$$
\begin{aligned}
& \int_{\mathbf{R}^{N}} v^{\otimes 2}: w(s, x) \otimes n_{x} F_{\epsilon}(s, x, v) d v \\
= & \alpha \int_{\mathbf{R}^{N}}(w(s, x) \cdot v)\left(v \cdot n_{x}\right)_{+} F_{\epsilon}(s, x, v) d v \\
& -\alpha \Lambda_{x}\left(\frac{F_{\epsilon}}{M}\right) \int_{\mathbf{R}^{N}}(w(s, x) \cdot v)\left(v \cdot n_{x}\right)+M d v,
\end{aligned}
$$


and the last integral vanishes since the integrand is odd in the tangential component of $v$.

Finally, whenever $w \in C_{c}^{1}\left(\mathbf{R}_{+} \times \bar{\Omega}\right)$ is tangential on $\partial \Omega$, one has

$$
\begin{aligned}
& \int_{\mathbf{R}^{N}} v^{\otimes 2}: w(s, x) \otimes n_{x} F_{\epsilon}(s, x, v) d v \\
= & \alpha \int_{\mathbf{R}^{N}}(w(s, x) \cdot v)\left(v \cdot n_{x}\right)_{+} F_{\epsilon}(s, x, v) d v .
\end{aligned}
$$

Therefore, each classical solution $F_{\epsilon} \in C\left(\mathbf{R}_{+} \times \bar{\Omega} \times \mathbf{R}^{N}\right)$ of the initial-boundary value problem (4.1)-(4.2)-(4.3) that is continuously differentiable in $(t, x)$ and satisfies (4.4) also satisfies

$$
\begin{aligned}
& \int_{0}^{t} \iint_{\Omega \times \mathbf{R}^{N}}\left(\epsilon v \cdot \partial_{t} w(s, x)+v^{\otimes 2}: \nabla_{x} w(s, x)\right) F_{\epsilon}(s, x, v) d v d x d s \\
= & \alpha \int_{0}^{t} \int_{\partial \Omega \times \mathbf{R}^{N}}(w(s, x) \cdot v)\left(v \cdot n_{x}\right)_{+} F_{\epsilon}(s, x, v) d v d S(x) d s \\
& +\epsilon \iint_{\Omega \times \mathbf{R}^{N}} w(t, x) \cdot v F_{\epsilon}(t, x, v) d v d x \\
& -\epsilon \iint_{\Omega \times \mathbf{R}^{N}} w(0, x) \cdot v F_{\epsilon}^{i n}(x, v) d v d x .
\end{aligned}
$$

Henceforth, we shall consider exclusively renormalized solutions of the initialboundary value problem (4.1)-(4.2)-(4.3) satisfying the identity (4.5) for each vector field $w \in C_{c}^{1}\left(\mathbf{R}_{+} \times \bar{\Omega}\right)$ tangential on $\partial \Omega$ and such that $\operatorname{div}_{x} w=0$ on $\Omega$.

Now we prove estimate d) in Theorem 4.1.

Proof. [Proof of estimate d).] A renormalized solution of the initial-boundary value problem (4.1)-(4.2)-(4.3) can be constructed as the limit for $\epsilon>0$ fixed and $m \rightarrow+\infty$ of solutions $F_{\epsilon, m}$ of the approximating equation

$$
\epsilon \partial_{t} F_{\epsilon, m}+v \cdot \nabla_{x} F_{\epsilon, m}=\frac{1}{\epsilon^{1+q}} \frac{\mathcal{B}_{m}\left(F_{\epsilon, m}, F_{\epsilon, m}\right)}{1+\frac{1}{m} \int_{\mathbf{R}^{N}} F_{\epsilon, m} d v}, \quad(x, v) \in \Omega \times \mathbf{R}^{N},
$$

with the same initial and boundary conditions (4.2)-(4.3) satisfied by $F_{\epsilon, m}$, where the approximate collision integral is given by the same expression as Boltzmann's collision integral with collision kernel $b$ replaced with its truncated variant $b_{m}$ defined as ${ }^{1}$

$$
b_{m}\left(v-v_{*}, \omega\right):=m \wedge b\left(v-v_{*}, \omega\right) .
$$

Let $\xi$ be a compactly supported $C^{1}$ vector field satisfying

$$
\xi(x)=a(x) n_{x} \text { for each } x \in \partial \Omega \text {, with } a \geq 0 \text { on } \partial \Omega \text { and } a=1 \text { on } K .
$$

Since the approximate collision integral in (4.6) is normalized with an average of $F_{\epsilon, m}$ with respect to $v$, all solutions of that equation satisfy equality (4.5) for any

\footnotetext{
${ }^{1}$ For all $x, y \in \mathbf{R}$, the notation $x \wedge y$ designates $\min (x, y)$.
} 
$w \in C_{c}^{1}\left(\mathbf{R}_{+} \times \mathbf{R}^{N}\right)$, i.e. the local conservation of momentum. In other words,

$$
\begin{aligned}
& \int_{0}^{t} \iint_{\Omega \times \mathbf{R}^{N}}\left(v^{\otimes 2}: \nabla_{x} \xi(x)\right) F_{\epsilon, m}(s, x, v) d v d x d s \\
= & \int_{0}^{t} \iint_{\partial \Omega \times \mathbf{R}^{N}}(v \cdot \xi(x))\left(v \cdot n_{x}\right) F_{\epsilon, m}(s, x, v) d v d S(x) d s \\
& +\epsilon \iint_{\Omega \times \mathbf{R}^{N}} \xi(x) \cdot v F_{\epsilon, m}(t, x, v) d v d x \\
& -\epsilon \iint_{\Omega \times \mathbf{R}^{N}} \xi(x) \cdot v F_{\epsilon, m}^{i n}(x, v) d v d x .
\end{aligned}
$$

Therefore, since $F_{\epsilon, m} \geq 0$ a.e. and $(v \cdot \xi(x))\left(v \cdot n_{x}\right)=a(x)\left(v \cdot n_{x}\right)^{2} \geq 0$ for each $x \in \partial \Omega$ and $v \in \mathbf{R}^{N}$, one has

$$
\begin{aligned}
0 \leq & \int_{0}^{t} \iint_{K \times \mathbf{R}^{N}}\left(v \cdot n_{x}\right)^{2} F_{\epsilon, m}(s, x, v) d v d S(x) d s \\
\leq & \int_{0}^{t} \iint_{\partial \Omega \times \mathbf{R}^{N}}(v \cdot \xi(x))\left(v \cdot n_{x}\right) F_{\epsilon, m}(s, x, v) d v d S(x) d s \\
= & \int_{0}^{t} \iint_{\Omega \times \mathbf{R}^{N}}\left(v^{\otimes 2}: \nabla_{x} \xi(x)\right) F_{\epsilon, m}(s, x, v) d v d x d s \\
& +\epsilon \iint_{\Omega \times \mathbf{R}^{N}} \xi(x) \cdot v F_{\epsilon, m}^{i n}(x, v) d v d x \\
& -\epsilon \iint_{\Omega \times \mathbf{R}^{N}} \xi(x) \cdot v F_{\epsilon, m}(t, x, v) d v d x .
\end{aligned}
$$

At this point, we recall that the function $h: z \mapsto(1+z) \ln (1+z)-z$ introduced in Theorem 4.1 has Legendre dual

$$
h^{*}(\zeta)=\sup _{z>-1}(\zeta z-h(z))=e^{\zeta}-\zeta-1 .
$$

By Young's inequality — or equivalently, by definition of $h^{*}$ (see for instance [5]) one has

$$
\frac{1}{4}\left(1+|v|^{2}\right) \epsilon\left|g_{\epsilon, n}\right| \leq h\left(\epsilon\left|g_{\epsilon, n}\right|\right)+h^{*}\left(\frac{1}{4}\left(1+|v|^{2}\right)\right)
$$

so that, for each nonnegative $\chi \in C_{c}\left(\mathbf{R}^{N}\right)$,

$$
\begin{aligned}
& \iint_{\Omega \times \mathbf{R}^{N}} \chi(x)\left(1+|v|^{2}\right) F_{\epsilon, m}(t, x, v) d v d x \\
= & \iint_{\Omega \times \mathbf{R}^{N}} \chi(x)\left(1+|v|^{2}\right)\left(1+\epsilon g_{\epsilon, m}\right)(t, x, v) M(v) d v d x \\
\leq & \iint_{\Omega \times \mathbf{R}^{N}} \chi(x)\left(1+h^{*}\left(\frac{1}{4}\left(1+|v|^{2}\right)\right)\right) M(v) d v d x \\
& +\|\chi\|_{L^{\infty}} H\left(F_{\epsilon, m} \mid M\right)(t) \\
\leq & \iint_{\Omega \times \mathbf{R}^{N}} \chi(x)\left(1+h^{*}\left(\frac{1}{4}\left(1+|v|^{2}\right)\right)\right) M(v) d v d x \\
& +\|\chi\|_{L^{\infty}} H\left(F_{\epsilon}^{i n} \mid M\right),
\end{aligned}
$$


since the relative entropy estimate c) in Theorem 4.1 is also satisfied by the approximate solution $F_{\epsilon, m}$.

Since $\xi$ is compactly supported, putting together (4.7), (4.8), and letting $m \rightarrow+\infty$ leads to estimate d).

4.2. The Euler limit. Let $u^{i n} \in \mathcal{H}(\Omega)$, and pick initial data $F_{\epsilon}^{i n}$ for the Boltzmann Equation satisfying

$$
\frac{1}{\epsilon^{2}} H\left(F_{\epsilon}^{i n} \mid \mathcal{M}_{1, \epsilon u^{i n}, 1}\right) \rightarrow 0 .
$$

TheOREM 4.2. For each $\epsilon>0$, let $F_{\epsilon}$ be a renormalized solution of (4.1)-(4.2)-(4.3) satisfying the local momentum conservation law (4.5) for each $w \in C_{c}^{1}\left(\mathbf{R}_{+} \times \bar{\Omega}\right)$ such that $\operatorname{div}_{x} w=0$ and $\left.w \cdot n_{x}\right|_{\partial \Omega}=0$. Assume that the accommodation parameter $\alpha$ in the accommodation condition (4.2) at the boundary depends on the scaling parameter $\epsilon$ in such a way that

$$
\alpha(\epsilon)=o(\epsilon) \text { as } \epsilon \rightarrow 0 .
$$

Then, for each compact $K \subset \bar{\Omega}$, the family

$$
\frac{1}{\epsilon} \int_{\mathbf{R}^{N}} v F_{\epsilon} d v
$$

is bounded in $L^{\infty}\left(\mathbf{R}_{+} ; L^{1}(K)\right)$ and weakly relatively compact in $L^{1}([0, T] \times K)$ for all $T>0$, and each of its limit points as $\epsilon \rightarrow 0$ is a dissipative solution of the Euler Equation (2.7).

Assume that $u^{i n} \in \mathcal{H}(\Omega)$ is smooth enough so that the initial-boundary value problem for the Euler Equation (2.7) has a classical solution on some finite time interval $[0, T]$ - for instance $u^{i n} \in H^{s}(\Omega)$ with $s>\frac{N}{2}+1$, or $u^{i n} \in C^{1, \theta}$ with $0<\theta<1$. In that case, the convergence result above can be strengthened with the notion of entropic convergence, invented by Dave Levermore specifically to handle such problems.

Definition 4.3 (C. Bardos, F. Golse, C.D. Levermore [5]). A family $g_{\epsilon} \equiv g_{\epsilon}(x, v)$ of $L_{l o c}^{1}\left(\Omega \times \mathbf{R}^{N} ; M d x d v\right)$ is said to converge entropically of order $\epsilon$ to $g \equiv g(x, v)$ as $\epsilon \rightarrow 0$ if the following conditions hold:

(i) $1+\epsilon g_{\epsilon} \geq 0$ a.e. on $\Omega \times \mathbf{R}^{N}$ for each $\epsilon$,

(ii) $g_{\epsilon} \rightarrow g$ weakly in $L_{l o c}^{1}\left(\Omega \times \mathbf{R}^{N} ; M d x d v\right)$ as $\epsilon \rightarrow 0$,

(iii)

$$
\frac{1}{\epsilon^{2}} H\left(M\left(1+\epsilon g_{\epsilon}\right) \mid M\right) \rightarrow \frac{1}{2} \iint_{\Omega \times \mathbf{R}^{N}} g(x, v)^{2} M(v) d x d v
$$

as $\epsilon \rightarrow 0$.

We recall that if $g_{\epsilon} \rightarrow g$ entropically of order $\epsilon$ then, for each compact $K \subset \Omega$, one has

$$
\int_{K} \int_{\mathbf{R}^{N}}\left(1+|v|^{2}\right)\left|g_{\epsilon}(x, v)-g(x, v)\right| M(v) d v d x \rightarrow 0 \quad \text { as } \epsilon \rightarrow 0 .
$$

In other words, entropic convergence implies strong $L^{1}$ convergence with the weight $\left(1+|v|^{2}\right) M(v)$ (see Proposition 4.11 in [5]). 
Whenever the incompressible Euler Equation (2.7) has a classical solution $u$ on $[0, T] \times \Omega$, using the weak-strong uniqueness property of dissipative solutions and the conservation of energy satisfied by classical solutions of (2.7) we arrive at the following stronger convergence result, which is a straightforward consequence of Theorem 4.2. The interested reader is referred to the proof of Theorem 6.2 in [5] where the squeezing argument leading from weak compactness to entropic convergence is explained in detail.

COROLlaRY 4.4. Consider a family $F_{\epsilon}^{i n} \equiv F_{\epsilon}^{i n}(x, v) \geq 0$ a.e. of measurable functions on $\Omega \times \mathbf{R}^{N}$ such that

$$
\frac{F_{\epsilon}^{i n}(x, v)-M(v)}{\epsilon M(v)} \rightarrow u^{i n}(x) \cdot v
$$

entropically of order $\epsilon$ as $\epsilon \rightarrow 0$, where $u^{\text {in }} \in \mathcal{H}(\Omega)$ is smooth enough so that the initialboundary value problem (2.7) has a classical solution $u$ defined on the time interval $[0, T]$ with $T>0$.

For each $\epsilon>0$, let $F_{\epsilon}$ be a renormalized solution of (4.1)-(4.2)-(4.3) satisfying the local momentum conservation law (4.5) for each $w \in C_{c}^{1}\left(\mathbf{R}_{+} \times \bar{\Omega}\right)$ that satisfies $\operatorname{div}_{x} w=0$ and $\left.w \cdot n_{x}\right|_{\partial \Omega}=0$. Assume that the accommodation parameter $\alpha$ in the accommodation condition (4.2) at the boundary depends on the scaling parameter $\epsilon$ in such a way that

$$
\alpha(\epsilon)=o(\epsilon) \text { as } \epsilon \rightarrow 0
$$

Then

$$
\frac{F_{\epsilon}(t, x, v)-M(v)}{\epsilon M(v)} \rightarrow u(t, x) \cdot v
$$

entropically of order $\epsilon$ as $\epsilon \rightarrow 0$, for a.e. $t \in[0, T]$.

The proof of Theorem 4.2 above occupies the remaining part of the present section.

4.3. The relative entropy inequality. Statement c) in Theorem 4.1 bears on the evolution of the relative entropy of the distribution $F_{\epsilon}$ with respect to the uniform Maxwellian $M=\mathcal{M}_{1,0,1}$. In the next proposition, we consider the evolution of the relative entropy of the distribution $F_{\epsilon}$ with respect to a local Maxwellian of the form $\mathcal{M}_{1, \epsilon w, 1}$, where $w$ is a solenoidal velocity field on $\Omega$ that is tangential to $\partial \Omega$.

Proposition 4.5. Let $w \in C_{c}^{1}\left(\mathbf{R}_{+} \times \bar{\Omega}\right)$ be such that

$$
\operatorname{div}_{x} w=0 \quad \text { and }\left.\quad w \cdot n\right|_{\partial \Omega}=0 .
$$

Then, for each $\epsilon>0$, renormalized solutions $F_{\epsilon}$ of the initial-boundary value problem (4.1)-(4.2)-(4.3) satisfying the momentum conservation identity (4.5) also satisfy the relative entropy inequality

$$
\begin{aligned}
& \frac{1}{\epsilon^{2}} H\left(F_{\epsilon} \mid \mathcal{M}_{1, \epsilon w, 1}\right)(t)-\frac{1}{\epsilon^{2}} H\left(F_{\epsilon}^{i n} \mid \mathcal{M}_{1, \epsilon w(0, \cdot), 1}\right) \\
\leq & -\frac{1}{\epsilon^{4+q}} \int_{0}^{t} \int_{\Omega} \mathcal{P}_{\epsilon}(s, x) d x d s-\frac{\alpha}{\epsilon^{3}} \int_{0}^{t} \int_{\partial \Omega} \mathcal{D G}_{\epsilon}(s, x) d x d s \\
& -\frac{1}{\epsilon^{2}} \int_{0}^{t} \iint_{\Omega \times \mathbf{R}^{N}}(v-\epsilon w(s, x))^{\otimes 2}: \nabla_{x} w(s, x) F_{\epsilon}(s, x, v) d x d v d s
\end{aligned}
$$




$$
\begin{aligned}
& -\frac{1}{\epsilon} \int_{0}^{t} \iint_{\Omega \times \mathbf{R}^{N}}(v-\epsilon w(s, x)) \cdot E(w)(s, x) F_{\epsilon}(s, x, v) d v d x d s \\
& +\frac{\alpha}{\epsilon^{2}} \int_{0}^{t} \int_{\partial \Omega \times \mathbf{R}^{N}}(w(s, x) \cdot v)\left(v \cdot n_{x}\right)+F_{\epsilon}(s, x, v) d v d S(x) d s
\end{aligned}
$$

for each $t>0$.

Proof. We begin with the straightforward identity

$$
\begin{aligned}
H\left(F_{\epsilon} \mid \mathcal{M}_{1, \epsilon w, 1}\right) & =H\left(F_{\epsilon} \mid M\right)+\iint_{\Omega \times \mathbf{R}^{N}} F_{\epsilon} \ln \left(\frac{M}{\mathcal{M}_{1, \epsilon w, 1}}\right) d x d v \\
& =H\left(F_{\epsilon} \mid M\right)+\iint_{\Omega \times \mathbf{R}^{N}} F_{\epsilon}\left(\frac{1}{2}|v-\epsilon w|^{2}-\frac{1}{2}|v|^{2}\right) d x d v \\
& =H\left(F_{\epsilon} \mid M\right)+\iint_{\Omega \times \mathbf{R}^{N}} F_{\epsilon}\left(\frac{1}{2} \epsilon^{2}|w|^{2}-\epsilon v \cdot w\right) d x d v .
\end{aligned}
$$

Thus

$$
\begin{aligned}
& H\left(F_{\epsilon} \mid \mathcal{M}_{1, \epsilon w, 1}\right)(t)-H\left(F_{\epsilon} \mid \mathcal{M}_{1, \epsilon w, 1}\right)(0) \\
= & H\left(F_{\epsilon} \mid M\right)(t)-H\left(F_{\epsilon} \mid M\right)(0)+\frac{1}{2} \epsilon^{2} \iint_{\Omega \times \mathbf{R}^{N}} F_{\epsilon}(t, x, v)|w(t, x)|^{2} d x d v \\
& -\frac{1}{2} \epsilon^{2} \iint_{\Omega \times \mathbf{R}^{N}} F_{\epsilon}^{i n}(x, v)|w(0, x)|^{2} d x d v-\epsilon \iint_{\Omega \times \mathbf{R}^{N}} F_{\epsilon}(t, x, v) v \cdot w(t, x) d x d v \\
& +\epsilon \iint_{\Omega \times \mathbf{R}^{N}} F_{\epsilon}^{i n}(x, v) v \cdot w(0, x) d x d v .
\end{aligned}
$$

According to the continuity equation in statement a) in Theorem 4.1,

$$
\begin{aligned}
& \frac{1}{2} \epsilon^{2} \iint_{\Omega \times \mathbf{R}^{N}} F_{\epsilon}(t, x, v)|w(t, x)|^{2} d x d v-\frac{1}{2} \epsilon^{2} \iint_{\Omega \times \mathbf{R}^{N}} F_{\epsilon}^{i n}(x, v)|w(0, x)|^{2} d x d v \\
= & \int_{0}^{t} \iint_{\Omega \times \mathbf{R}^{N}} F_{\epsilon}\left(\epsilon^{2} \partial_{t}+\epsilon v \cdot \nabla_{x}\right) \frac{1}{2}|w|^{2} d x d v d s \\
= & \int_{0}^{t} \iint_{\Omega \times \mathbf{R}^{N}} F_{\epsilon} w \cdot\left(\epsilon^{2} \partial_{t} w+\epsilon v \cdot \nabla_{x} w\right) d x d v d s .
\end{aligned}
$$

In (4.11), we replace the term $H\left(F_{\epsilon} \mid M\right)(t)-H\left(F_{\epsilon} \mid M\right)(0)$ with the right hand side of the inequality of statement c) of Theorem 4.1, and use (4.12) together with (4.5) to arrive at

$$
\begin{aligned}
& H\left(F_{\epsilon} \mid \mathcal{M}_{1, \epsilon w, 1}\right)(t)-H\left(F_{\epsilon} \mid \mathcal{M}_{1, \epsilon w, 1}\right)(0) \\
\leq & -\frac{1}{\epsilon^{2+q}} \int_{0}^{t} \int_{\Omega} \mathcal{P}_{\epsilon}(s, x) d x d s-\frac{\alpha}{\epsilon} \int_{0}^{t} \int_{\partial \Omega} \mathcal{D G}_{\epsilon}(s, x) d x d s \\
& +\int_{0}^{t} \iint_{\Omega \times \mathbf{R}^{N}} w \cdot\left(\epsilon^{2} \partial_{t} w+\epsilon v \cdot \nabla_{x} w\right)(s, x) F_{\epsilon}(s, x, v) d x d v d s \\
& -\int_{0}^{t} \iint_{\Omega \times \mathbf{R}^{N}}\left(\epsilon v \cdot \partial_{t} w(s, x)+v^{\otimes 2}: \nabla_{x} w(s, x)\right) F_{\epsilon}(s, x, v) d v d x d s \\
& +\alpha \int_{0}^{t} \int_{\partial \Omega \times \mathbf{R}^{N}}(w(s, x) \cdot v)\left(v \cdot n_{x}\right)_{+} F_{\epsilon}(s, x, v) d v d S(x) d s .
\end{aligned}
$$


Next we express $\partial_{t} w$ in terms of $E(w)=\partial_{t} w+w \cdot \nabla_{x} w$ and $\nabla_{x} w$ :

$$
\begin{aligned}
& \left(\epsilon v \cdot \partial_{t} w(s, x)+v^{\otimes 2}: \nabla_{x} w(s, x)\right)-w \cdot\left(\epsilon^{2} \partial_{t} w+\epsilon v \cdot \nabla_{x} w\right) \\
= & (v-\epsilon w)^{\otimes 2}: \nabla_{x} w+\epsilon(v-\epsilon w) \cdot E(w) .
\end{aligned}
$$

In the right hand side of (4.13), we substitute

$$
\begin{aligned}
& \int_{0}^{t} \iint_{\Omega \times \mathbf{R}^{N}} w \cdot\left(\epsilon^{2} \partial_{t} w+\epsilon v \cdot \nabla_{x} w\right)(s, x) F_{\epsilon}(s, x, v) d x d v d s \\
& -\int_{0}^{t} \iint_{\Omega \times \mathbf{R}^{N}}\left(\epsilon v \cdot \partial_{t} w(s, x)+v^{\otimes 2}: \nabla_{x} w(s, x)\right) F_{\epsilon}(s, x, v) d v d x d s \\
= & -\int_{0}^{t} \iint_{\Omega \times \mathbf{R}^{N}}(v-\epsilon w(s, x))^{\otimes 2}: \nabla_{x} w(s, x) F_{\epsilon}(s, x, v) d x d v d s \\
& -\epsilon \int_{0}^{t} \iint_{\Omega \times \mathbf{R}^{N}}(v-\epsilon w(s, x)) \cdot E(w)(s, x) F_{\epsilon}(s, x, v) d v d x d s
\end{aligned}
$$

and arrive at the relative entropy inequality (4.10).

4.4. Control of the boundary term. The relative entropy inequality (4.10) is the same as in the one considered in [10], [25], and [29], except for the boundary term - i.e. the last term on the right hand side, which is in general not nonpositive. Since the effect of the boundary is our main interest in this paper, and is the only difference with the case of the Cauchy problem treated in [29], the core of our argument is to obtain a control of that term.

LEMmA 4.6. With the notations of Theorem 4.1, for each $\epsilon>0$ and each divergencefree test vector field $w \in C_{c}^{1}\left(\mathbf{R}_{+} \times \bar{\Omega}\right)$ that is tangential on the boundary $\partial \Omega$, each renormalized solution $F_{\epsilon}$ of the initial-boundary value problem (4.1)-(4.2)-(4.3) satisfies the inequality

$$
\begin{aligned}
& \frac{\alpha}{\epsilon^{2}} \int_{\mathbf{R}^{N}}(w(s, x) \cdot v)\left(v \cdot n_{x}\right)_{+} F_{\epsilon}(s, x, v) d v \\
= & \frac{\alpha}{\epsilon} \int_{\mathbf{R}^{N}}(w(s, x) \cdot v)\left(v \cdot n_{x}\right)_{+} g_{\epsilon}(s, x, v) M(v) d v \\
\leq & \frac{\alpha}{2 \epsilon^{3}} \mathcal{D} \mathcal{G}_{\epsilon}(s, x)+\frac{\alpha}{\epsilon} C(w) \mathbf{1}_{w(s, x) \neq 0} \Lambda_{x}\left(F_{\epsilon} / M\right)(s, x)
\end{aligned}
$$

a.e. in $(s, x) \in \mathbf{R}_{+} \times \partial \Omega$, where

$$
C(w):=\frac{1}{2} \int_{\mathbf{R}^{N}}\left(e^{2\|w\|_{L^{\infty}}|v|}-2\|w\|_{L^{\infty}}|v|-1\right)\left(v_{1}\right)_{+} M(v) d v .
$$

We use Young's inequality for a translate of the function $h$ defined in Theorem 4.1 , much in the same way as in the proof of Theorem 6.2 in [5] (see especially pp. 738-739 there).

Proof. Let $z_{0}>-1$; for each $z>-1$, set

$$
l\left(z-z_{0}\right):=h(z)-h\left(z_{0}\right)-h^{\prime}\left(z_{0}\right)\left(z-z_{0}\right) .
$$

We recall that the Legendre dual of the function $h$ defined in Theorem 4.1 is

$$
h^{*}(p):=\sup _{z>-1}(p z-h(z))=e^{p}-p-1, \quad p \in \mathbf{R} .
$$


A straightforward computation shows that

$$
\begin{aligned}
l^{*}(p)= & \sup _{z>-1}\left(p\left(z-z_{0}\right)-l\left(z-z_{0}\right)\right) \\
= & \sup _{z>-1}\left(p\left(z-z_{0}\right)-h(z)+h\left(z_{0}\right)+h^{\prime}\left(z_{0}\right)\left(z-z_{0}\right)\right) \\
= & h\left(z_{0}\right)-\left(h^{\prime}\left(z_{0}\right)+p\right) z_{0}+\sup _{z>-1}\left(\left(h^{\prime}\left(z_{0}\right)+p\right) z-h(z)\right) \\
= & h\left(z_{0}\right)-\left(h^{\prime}\left(z_{0}\right)+p\right) z_{0}+h^{*}\left(h^{\prime}\left(z_{0}\right)+p\right) \\
= & h\left(z_{0}\right)-\left(h^{\prime}\left(z_{0}\right)+p\right) z_{0}+e^{h^{\prime}\left(z_{0}\right)} e^{p}-h^{\prime}\left(z_{0}\right)-p-1 \\
= & \left(1+z_{0}\right) \ln \left(1+z_{0}\right)-z_{0}-\left(\ln \left(1+z_{0}\right)+p\right) z_{0} \\
& +\left(1+z_{0}\right) e^{p}-\ln \left(1+z_{0}\right)-p-1 \\
= & \left(1+z_{0}\right)\left(e^{p}-p-1\right)=\left(1+z_{0}\right) h^{*}(p) .
\end{aligned}
$$

Writing $F_{\epsilon}=M\left(1+\epsilon g_{\epsilon}\right)$ and observing that

$$
\int_{\mathbf{R}^{N}}(w(s, x) \cdot v)\left(v \cdot n_{x}\right)_{+} M(v) d v=0, \quad(s, x) \in \mathbf{R}_{+} \times \partial \Omega
$$

since $w$ is tangential on $\partial \Omega$, one has

$$
\begin{aligned}
& \int_{\mathbf{R}^{N}}(w(s, x) \cdot v)\left(v \cdot n_{x}\right)_{+} F_{\epsilon}(s, x, v) d v \\
= & \epsilon \int_{\mathbf{R}^{N}}(w(s, x) \cdot v)\left(v \cdot n_{x}\right)_{+} g_{\epsilon}(s, x, v) M(v) d v .
\end{aligned}
$$

Then

$$
\begin{aligned}
& \int_{\mathbf{R}^{N}}(w(s, x) \cdot v)\left(v \cdot n_{x}\right)_{+} g_{\epsilon}(s, x, v) M(v) d v \\
= & \int_{\mathbf{R}^{N}}(w(s, x) \cdot v)\left(v \cdot n_{x}\right)_{+}\left(g_{\epsilon}(s, x, v)-\Lambda_{x}\left(g_{\epsilon}\right)\right) M(v) d v
\end{aligned}
$$

by (4.15) since $\Lambda_{x}\left(g_{\epsilon}\right)$ is independent of $v$.

By Young's inequality (or equivalently, by the definition of the Legendre dual of $l)$,

$$
2 \epsilon^{2}\left(g_{\epsilon}-\Lambda_{x}\left(g_{\epsilon}\right)\right)(w \cdot v) \leq l\left(\epsilon\left(g_{\epsilon}-\Lambda_{x}\left(g_{\epsilon}\right)\right)\right)+l^{*}(\epsilon(2 w \cdot v))
$$

so that

$$
\begin{aligned}
2 \int_{\mathbf{R}^{N}}(w \cdot v)\left(v \cdot n_{x}\right)_{+} g_{\epsilon} M d v \leq & \frac{1}{\epsilon^{2}} \int_{\mathbf{R}^{N}} l\left(\epsilon\left(g_{\epsilon}-\Lambda_{x}(\epsilon)\right)\right)\left(v \cdot n_{x}\right)_{+} M d v \\
& +\frac{1}{\epsilon^{2}} \int_{\mathbf{R}^{N}} l^{*}(\epsilon(2 w \cdot v))\left(v \cdot n_{x}\right)_{+} M d v
\end{aligned}
$$

First, since $\Lambda_{x}$ is the average under a probability measure, one has

$$
\begin{aligned}
& \int_{\mathbf{R}^{N}} l\left(\epsilon\left(g_{\epsilon}-\Lambda_{x}\left(g_{\epsilon}\right)\right)\right)\left(v \cdot n_{x}\right)_{+} M d v \\
= & \frac{1}{\sqrt{2 \pi}} \Lambda_{x}\left(l\left(\epsilon\left(g_{\epsilon}-\Lambda_{x}(\epsilon)\right)\right)\right) \\
= & \frac{1}{\sqrt{2 \pi}} \Lambda_{x}\left(h\left(\epsilon g_{\epsilon}\right)-h\left(\epsilon \Lambda_{x}\left(g_{\epsilon}\right)\right)-h^{\prime}\left(\epsilon \Lambda_{x}\left(g_{\epsilon}\right)\right)\left(\epsilon g_{\epsilon}-\epsilon \Lambda_{x}\left(g_{\epsilon}\right)\right)\right) \\
= & \frac{1}{\sqrt{2 \pi}} \Lambda_{x}\left(h\left(\epsilon g_{\epsilon}\right)-h\left(\epsilon \Lambda_{x}\left(g_{\epsilon}\right)\right)\right)-h^{\prime}\left(\epsilon \Lambda_{x}\left(g_{\epsilon}\right)\right) \frac{1}{\sqrt{2 \pi}} \Lambda_{x}\left(\epsilon g_{\epsilon}-\epsilon \Lambda_{x}\left(g_{\epsilon}\right)\right) \\
= & \frac{1}{\sqrt{2 \pi}}\left(\Lambda_{x}\left(h\left(\epsilon g_{\epsilon}\right)-h\left(\epsilon \Lambda_{x}\left(g_{\epsilon}\right)\right)\right)\right)=\mathcal{D} \mathcal{G}_{\epsilon} .
\end{aligned}
$$


On the other hand

$$
\begin{aligned}
& \frac{1}{\epsilon^{2}} \int_{\mathbf{R}^{N}} l^{*}(\epsilon(2 w \cdot v))\left(v \cdot n_{x}\right)_{+} M d v \\
= & \left(1+\epsilon \Lambda_{x}\left(g_{\epsilon}\right)\right) \int_{\mathbf{R}^{N}} \frac{e^{2 \epsilon|w||v|}-2 \epsilon|w||v|-1}{\epsilon^{2}}\left(v \cdot n_{x}\right)_{+} M d v \\
\leq & \Lambda_{x}\left(F_{\epsilon} / M\right) \int_{\mathbf{R}^{N}}\left(e^{2|w||v|}-2|w||v|-1\right)\left(v \cdot n_{x}\right)_{+} M d v
\end{aligned}
$$

since, for each $a>0$, the map

$$
\epsilon \mapsto \frac{e^{a \epsilon}-a \epsilon-1}{\epsilon^{2}}=a^{2} \sum_{n \geq 2} \frac{(a \epsilon)^{n-2}}{n !}
$$

is increasing.

Finally,

$$
\begin{aligned}
& \frac{\alpha}{\epsilon^{2}} \int_{\mathbf{R}^{N}}(w(s, x) \cdot v)\left(v \cdot n_{x}\right)_{+} F_{\epsilon}(s, x, v) d v \\
\leq & \frac{\alpha}{\epsilon} \int_{\mathbf{R}^{N}}(w(s, x) \cdot v)\left(v \cdot n_{x}\right)_{+} g_{\epsilon}(s, x, v) M(v) d v \\
\leq & \frac{\alpha}{2 \epsilon^{3}} \mathcal{D} \mathcal{G}_{\epsilon}(s, x)+\frac{\alpha}{\epsilon} C(w) \mathbf{1}_{w(s, x) \neq 0} \Lambda_{x}\left(F_{\epsilon} / M\right)(s, x),
\end{aligned}
$$

where

$$
C(w):=\frac{1}{2} \int_{\mathbf{R}^{N}}\left(e^{2\|w\|_{L^{\infty}}|v|}-2\|w\|_{L^{\infty}}|v|-1\right)\left(v_{1}\right)_{+} M(v) d v .
$$

After integrating both sides of (4.14) with respect to $(s, x)$, the first term on the right hand side of (4.14) will be absorbed by the Darrozes-Guiraud information on the right hand side of (4.10), so that, with Lemma 4.6, the inequality (4.10) is transformed into

$$
\begin{aligned}
& \frac{1}{\epsilon^{2}} H\left(F_{\epsilon} \mid \mathcal{M}_{1, \epsilon w, 1}\right)(t)-\frac{1}{\epsilon^{2}} H\left(F_{\epsilon}^{i n} \mid \mathcal{M}_{1, \epsilon w(0, \cdot), 1}\right) \\
\leq & -\frac{1}{\epsilon^{4+q}} \int_{0}^{t} \int_{\Omega} \mathcal{P}_{\epsilon}(s, x) d x d s-\frac{\alpha}{2 \epsilon^{3}} \int_{0}^{t} \int_{\partial \Omega} \mathcal{D G}_{\epsilon}(s, x) d x d s \\
& -\frac{1}{\epsilon^{2}} \int_{0}^{t} \iint_{\Omega \times \mathbf{R}^{N}}(v-\epsilon w(s, x))^{\otimes 2}: \nabla_{x} w(s, x) F_{\epsilon}(s, x, v) d x d v d s \\
& -\frac{1}{\epsilon} \int_{0}^{t} \iint_{\Omega \times \mathbf{R}^{N}}(v-\epsilon w(s, x)) \cdot E(w)(s, x) F_{\epsilon}(s, x, v) d v d x d s \\
& +\frac{\alpha}{\epsilon} C(w) \int_{0}^{t} \int_{\partial \Omega \cap \operatorname{supp}(w)} \Lambda_{x}\left(F_{\epsilon} / M\right)(s, x) d S(x) d s,
\end{aligned}
$$

for all $t \geq 0$.

4.5. Control of the outgoing mass flux. In the lemma below, we shall prove that the outgoing mass flux $\Lambda_{x}\left(F_{\epsilon}\right)$ is uniformly bounded in $L_{l o c}^{1}\left(\mathbf{R}_{+} \times \partial \Omega\right)$, so that the last term on the right hand side of (4.16) vanishes under the assumption $\alpha(\epsilon)=o(\epsilon)$ as $\epsilon \rightarrow 0$. 
LEMMA 4.7. With the notations of Theorem 4.1, for each $\epsilon>0$, each renormalized solution $F_{\epsilon}$ of the initial-boundary value problem (4.1)-(4.2)-(4.3) satisfies the inequality

$$
\begin{aligned}
\int_{\mathbf{R}^{N}} F_{\epsilon}(t, x, v)\left(v \cdot n_{x}\right)_{+} d v \leq & \frac{C_{1}}{\sqrt{2 \pi} h(\eta)} \mathcal{D} \mathcal{G}_{\epsilon}(t, x) \\
& +\frac{C_{1}}{\sqrt{2 \pi}(1-\eta)} \int_{\mathbf{R}^{N}} F_{\epsilon}\left(v \cdot n_{x}\right)^{2} d v
\end{aligned}
$$

for all $\eta \in(0,1)$, a.e. in $(t, x) \in \mathbf{R}_{+} \times \partial \Omega$, where

$$
C_{1}=\left(\int_{0}^{\infty}\left(z^{2} \wedge 1\right) e^{-z^{2} / 2} \frac{d z}{\sqrt{2 \pi}}\right)^{-1}
$$

Proof. First we recast the Darrozes-Guiraud information in the form

$$
\begin{aligned}
\mathcal{D} \mathcal{G}_{\epsilon} & =\frac{1}{\sqrt{2 \pi}} \Lambda_{x}\left(h\left(\epsilon g_{\epsilon}\right)-h\left(\epsilon \Lambda_{x}\left(g_{\epsilon}\right)\right)\right) \\
& =\frac{1}{\sqrt{2 \pi}} \Lambda_{x}\left(G_{\epsilon} \ln G_{\epsilon}-G_{\epsilon}-\Lambda_{x}\left(G_{\epsilon}\right) \ln \Lambda_{x}\left(G_{\epsilon}\right)+\Lambda_{x}\left(G_{\epsilon}\right)\right) \\
& =\frac{1}{\sqrt{2 \pi}} \Lambda_{x}\left(G_{\epsilon} \ln \left(\frac{G_{\epsilon}}{\Lambda_{x}\left(G_{\epsilon}\right)}\right)-G_{\epsilon}+\Lambda_{x}\left(G_{\epsilon}\right)\right)
\end{aligned}
$$

since

$$
\Lambda_{x}\left(\left(G_{\epsilon}-\Lambda_{x}\left(G_{\epsilon}\right)\right) \ln \Lambda_{x}\left(G_{\epsilon}\right)\right)=\Lambda_{x}\left(\left(G_{\epsilon}-\Lambda_{x}\left(G_{\epsilon}\right)\right)\right) \ln \Lambda_{x}\left(G_{\epsilon}\right)=0 .
$$

Then we consider the integral

$$
\begin{aligned}
I:= & \Lambda_{x} G_{\epsilon} \int_{\mathbf{R}^{N}}\left(v \cdot n_{x}\right)_{+}^{2} \wedge 1 M d v \\
= & \Lambda_{x} G_{\epsilon} \int_{\mathbf{R}^{N}} \mathbf{1}_{\left|G_{\epsilon} / \Lambda_{x} G_{\epsilon}-1\right|>\eta}\left(v \cdot n_{x}\right)_{+}^{2} \wedge 1 M d v \\
& +\Lambda_{x} G_{\epsilon} \int_{\mathbf{R}^{N}} \mathbf{1}_{\left|G_{\epsilon} / \Lambda_{x} G_{\epsilon}-1\right| \leq \eta}\left(v \cdot n_{x}\right)_{+}^{2} \wedge 1 M d v=: I_{1}+I_{2}
\end{aligned}
$$

with $\eta \in] 0,1[$.

The first term is estimated in terms of the Darrozes-Guiraud information on the boundary, in view of (4.18):

$$
\begin{aligned}
I_{1} & \leq \frac{1}{h(\eta)} \Lambda_{x} G_{\epsilon} \int_{\mathbf{R}^{N}} \mathbf{1}_{\left|G_{\epsilon} / \Lambda_{x} G_{\epsilon}-1\right|>\eta} h\left(\left|\frac{G_{\epsilon}}{\Lambda_{x} G_{\epsilon}}-1\right|\right)\left(v \cdot n_{x}\right)_{+}^{2} \wedge 1 M d v \\
& \leq \frac{1}{h(\eta)} \Lambda_{x} G_{\epsilon} \int_{\mathbf{R}^{N}} h\left(\frac{G_{\epsilon}}{\Lambda_{x} G_{\epsilon}}-1\right)\left(v \cdot n_{x}\right)_{+}^{2} \wedge 1 M d v \\
& \leq \frac{1}{h(\eta)} \int_{\mathbf{R}^{N}}\left(G_{\epsilon} \ln \left(\frac{G_{\epsilon}}{\Lambda_{x} G_{\epsilon}}\right)-G_{\epsilon}+\Lambda_{x} G_{\epsilon}\right)\left(v \cdot n_{x}\right)_{+} M d v \\
& \leq \frac{1}{h(\eta)} \mathcal{D G}_{\epsilon}(t, x) .
\end{aligned}
$$

As for the second term, one has

$$
\begin{aligned}
I_{2} & =\Lambda_{x} G_{\epsilon} \int_{\mathbf{R}^{N}} \mathbf{1}_{\left|G_{\epsilon} / \Lambda_{x} G_{\epsilon}-1\right| \leq \eta}\left(v \cdot n_{x}\right)_{+}^{2} \wedge 1 M d v \\
& \leq \frac{1}{1-\eta} \int_{\mathbf{R}^{N}} G_{\epsilon} \mathbf{1}_{\left|G_{\epsilon} / \Lambda_{x} G_{\epsilon}-1\right| \leq \eta}\left(v \cdot n_{x}\right)_{+}^{2} \wedge 1 M d v \\
& \leq \frac{1}{1-\eta} \int_{\mathbf{R}^{N}} G_{\epsilon}\left(v \cdot n_{x}\right)^{2} M d v .
\end{aligned}
$$


Combining both estimates gives

$$
I=I_{1}+I_{2} \leq \frac{1}{h(\eta)} \mathcal{D} \mathcal{G}_{\epsilon}(t, x)+\frac{1}{1-\eta} \int_{\mathbf{R}^{N}} F_{\epsilon}\left(v \cdot n_{x}\right)^{2} d v .
$$

Since

$$
\int_{\mathbf{R}^{N}}\left(v \cdot n_{x}\right)_{+}^{2} \wedge 1 M d v=\int_{0}^{\infty} z^{2} \wedge 1 e^{-z^{2} / 2} \frac{d z}{\sqrt{2 \pi}}=: \frac{1}{C_{1}}>0
$$

is independent of $x$, we conclude that

$$
\int_{\mathbf{R}^{N}} F_{\epsilon}(t, x, v)\left(v \cdot n_{x}\right)_{+} d v=\frac{1}{\sqrt{2 \pi}} \Lambda_{x}\left(G_{\epsilon}\right)(t, x)=\frac{C_{1}}{\sqrt{2 \pi}} I
$$

which, together with the previous inequality, leads to the announced estimate.

4.6. Convergence to the incompressible Euler Equations. At this point we bring together the relative entropy inequality (4.10) and the boundary control (4.14), thereby arriving at the estimate

$$
\begin{aligned}
& \frac{1}{\epsilon^{2}} H\left(F_{\epsilon} \mid \mathcal{M}_{1, \epsilon w, 1}\right)(t)-\frac{1}{\epsilon^{2}} H\left(F_{\epsilon}^{i n} \mid \mathcal{M}_{1, \epsilon w(0, \cdot), 1}\right) \\
\leq & -\frac{1}{\epsilon^{4+q}} \int_{0}^{t} \int_{\Omega} \mathcal{P}_{\epsilon}(s, x) d x d s-\frac{\alpha}{2 \epsilon^{3}} \int_{0}^{t} \int_{\partial \Omega} \mathcal{D} \mathcal{G}_{\epsilon}(s, x) d x d s \\
& -\frac{1}{\epsilon^{2}} \int_{0}^{t} \iint_{\Omega \times \mathbf{R}^{N}}(v-\epsilon w(s, x))^{\otimes 2}: \nabla_{x} w(s, x) F_{\epsilon}(s, x, v) d x d v d s \\
& -\frac{1}{\epsilon} \int_{0}^{t} \iint_{\Omega \times \mathbf{R}^{N}}(v-\epsilon w(s, x)) \cdot E(w)(s, x) F_{\epsilon}(s, x, v) d v d x d s \\
& +\frac{\alpha}{\epsilon} C(w) \int_{0}^{t} \int_{\partial \Omega \cap \operatorname{supp}(w)} \Lambda_{x}\left(F_{\epsilon} / M\right)(s, x) d S(x) d s .
\end{aligned}
$$

Next, we use the pointwise inequality (4.17) with, say, $\eta=\frac{1}{2}$, to control the last integral on the right-hand side above:

$$
\begin{aligned}
& \frac{1}{\epsilon^{2}} H\left(F_{\epsilon} \mid \mathcal{M}_{1, \epsilon w, 1}\right)(t)-\frac{1}{\epsilon^{2}} H\left(F_{\epsilon}^{i n} \mid \mathcal{M}_{1, \epsilon w(0, \cdot), 1}\right) \\
\leq & -\frac{1}{\epsilon^{4+q}} \int_{0}^{t} \int_{\Omega} \mathcal{P}_{\epsilon}(s, x) d x d s \\
& -\frac{\alpha}{2 \epsilon^{3}}\left(1-\frac{2 C_{1}}{h(1 / 2)} C(w) \epsilon\right) \int_{0}^{t} \int_{\partial \Omega} \mathcal{D} \mathcal{G}_{\epsilon}(s, x) d x d s \\
& -\frac{1}{\epsilon^{2}} \int_{0}^{t} \iint_{\Omega \times \mathbf{R}^{N}}(v-\epsilon w(s, x))^{\otimes 2}: \nabla_{x} w(s, x) F_{\epsilon}(s, x, v) d x d v d s \\
& -\frac{1}{\epsilon} \int_{0}^{t} \iint_{\Omega \times \mathbf{R}^{N}}(v-\epsilon w(s, x)) \cdot E(w)(s, x) F_{\epsilon}(s, x, v) d v d x d s \\
& +\frac{2 \alpha}{\epsilon} C_{1} C(w) \int_{0}^{t} \int_{\partial \Omega \cap \operatorname{supp}(w)} \int_{\mathbf{R}^{N}} F_{\epsilon}\left(v \cdot n_{x}\right)^{2} d v d S(x) d s .
\end{aligned}
$$

Now, statement d) in Theorem 4.1 and the scaling assumption on the accommodation parameter, i.e. $\alpha(\epsilon)=o(\epsilon)$, show that, for each $T>0$,

$$
\frac{2 \alpha}{\epsilon} \int_{0}^{t} \int_{\partial \Omega \cap \operatorname{supp}(w)} \int_{\mathbf{R}^{N}} F_{\epsilon}\left(v \cdot n_{x}\right)^{2} d v d S(x) d s \rightarrow 0
$$


uniformly in $t \in[0, T]$ as $\epsilon \rightarrow 0$.

With (4.20), the relative entropy inequality (4.19) is precisely of the same form as the inequality stated as Theorem 5 in [29]. One then concludes by the same argument as in [29].

\section{Conclusion and final remarks}

As recalled above, the convergence of solutions of the boundary value problem for the Navier-Stokes Equations in the large Reynolds number regime is an open problem, as well as the validity of the Prandlt Equation for the boundary layer. Convergence to solutions of the Euler Equation, for general boundary conditions (including the Dirichlet boundary condition) are proved under only the most stringent regularity assumptions.

On the other hand the onset of von Karman vortex streets and the Kolmogorov hypothesis on turbulence based on a non zero energy dissipation in the large Reynolds regime (see chapter 5 in [13]) suggest that, in general, the limit is not a solution of the Euler Equation. This is in agreement with Kato's criterion [19] relating the convergence of the solutions of the Navier-Stokes Equations with Dirichlet boundary condition to a solution of the Euler Equations with the vanishing of the viscous energy dissipation in a boundary layer with thickness $O\left(\mathrm{Re}^{-1}\right)$.

For the Navier slip boundary condition (2.13) or (2.12), the inviscid limit established in the present paper is proved under the sole assumption $\lambda \rightarrow 0$ as $\nu \rightarrow 0$.

The following remarks are in order:

- at variance with previous results $[8,37]$, no regularity assumption is required for all the results in the present paper, as only energy estimates are used in the proof;

- what is proved here is the convergence to a dissipative solution (hence to the unique classical solution whenever it exists); therefore, this convergence is also true even if no classical solution exists, or even if the $L^{2}$ initial data corresponds to a "wild" solution à la C. DeLellis and L. Szkelyhidi (see [11]) for which there is no uniqueness of the Euler solution;

- therefore the main goal of the present paper is to show the strong similarity between the inviscid limit for the Navier-Stokes Equations and the fluid dynamic limit for the Boltzmann Equation; the accommodation coefficient $\alpha$, the Mach Ma and Strouhal Sh numbers, the Reynolds number Re (see [35], $\S 1.9)$, and the slip coefficient $\lambda$ are related by

$$
\mathrm{Ma}=\mathrm{Sh}=\epsilon, \quad \frac{1}{\mathrm{Re}}=o(1), \quad \frac{\alpha}{\epsilon}=\lambda,
$$

as $\epsilon \rightarrow 0$, so that the conditions $\alpha=o(\epsilon)$ as $\epsilon \rightarrow 0$ and $\lambda \rightarrow 0$ as $\nu \rightarrow 0$ are consistent.

In any case the convergence of the solution of the Navier-Stokes Equations to a classical solution of the Euler Equations implies that the energy dissipation vanishes in the limit, as observed by Kato [19]. Likewise, the entropic convergence obtained in the present paper in the case of renormalized solutions of the Boltzmann Equation implies that the sum of the entropy dissipation and of the Darrozes-Guiraud information at the boundary vanishes with $\epsilon$.

By analogy with the work of Kato [19], a possible conjecture is that, whenever

$$
\varliminf_{\epsilon \rightarrow 0} \frac{\alpha(\epsilon)}{\epsilon}>0,
$$


the vanishing of both the Darrozes-Guiraud information at the boundary and of the entropy production implies that the inviscid fluid dynamic limit of the Boltzmann Equation is described by a solution of the Euler Equations.

Other, perhaps less delicate problems could be analyzed by the methods used in the present paper. For instance, Maxwell's accommodation is but one example in a wide class of nonlocal boundary conditions for the Boltzmann Equation; the Navier-Stokes and Euler hydrodynamic limits of the Boltzmann Equation should be considered also for such boundary conditions (see [35] §1.6). Likewise, the condition (4.5), which may not be satisfied for all renormalized solutions of the initial boundary value problem for the Boltzmann Equation, should be removed on principle (as in [29]).

In other words, there remain many open problems related to the issues discussed in the present paper, to which we shall return in future publications.

Acknowledgment. Part of this work was completed while the first two authors were participating to the program "Partial Differential Equations in Kinetic Theories" hosted by the Isaac Newton Institute for Mathematical Sciences in Cambridge. We are pleased to thank the Institute for its generous hospitality and support.

\section{REFERENCES}

[1] K. Aoki, T. Inamuro, and Y. Onishi, Slightly rarefied gas flow over a body with small accommodation coefficient, J. Phys. Soc. Japan, 47, 663-671, 1997.

[2] C. Bardos, Existence et unicité de la solution de l'équation d'Euler en dimension deux, J. Math. Anal. Appl., 40, 769-790, 1972.

[3] C. Bardos, F. Golse, and C.D. Levermore, Sur les limites asymptotiques de la théorie cinétique conduisant à la dynamique des fluides incompressibles, C.R. Acad. Sci. Paris Sér. I, 309, 727-732, 1989.

[4] C. Bardos, F. Golse, and C.D. Levermore, Fluid dynamic limits of the Boltzmann equation I, J. Stat. Phys., 63, 323-344, 1991.

[5] C. Bardos, F. Golse, and C.D. Levermore, Fluid dynamic limits of Kinetic equations II: Convergence proofs for the Boltzmann equation, Commun. Pure and Appl. Math., 46, 667-753, 1993.

[6] C. Bardos and E. Titi, Euler equations for incompressible ideal fluids, Russian Math. Surveys, 62, 1-43, 2007.

[7] H. Beirão da Veiga, Regularity for Stokes and generalized Stokes systems under nonhomogeneous slip-type boundary conditions, Adv. Differ. Eqs., 9, 1079-1114, 2004.

[8] H. Beirão da Veiga and F. Crispo, Sharp inviscid limit results under Navier type boundary conditions. An $L^{p}$ theory, J. Math. Fluid Mech., 12, 397-411, 2010.

[9] H. Beirão da Veiga and F. Crispo, A missed persistence property for the Euler equations, and its effect on inviscid limits, preprint, arXiv 1011:1117v.

[10] F. Bouchut, F. Golse, and M. Pulvirenti, Kinetic Equations and Asymptotic Theory, L. Desvillettes \& B. Perthame (ed.), Editions scientifiques et médicales Elsevier, Paris, 2000.

[11] C. De Lellis and L. Szkelyhidi Jr, The Euler equations as a differential inclusion, Ann. of Math., (2)170, 1417-1436, 2009.

[12] R.J. DiPerna and P.L. Lions, On the Cauchy problem for the Boltzmann equation: Global existence and weak stability results, Ann. of Math., 130, 321-366, 1990.

[13] U. Frisch, Turbulence: The Legacy of A.N. Kolmogorov, Cambridge University Press, Cambridge, 1995.

[14] F. Golse and C.D. Levermore, The Stokes-Fourier and acoustic limits for the Boltzmann equation, Commun. Pure Appl. Math., 55, 336-393, 2002.

[15] F. Golse and L. Saint-Raymond, The Navier-Stokes limit of the Boltzmann equation for bounded collision kernels, Invent. Math., 155, 81-161, 2004.

[16] F. Golse and L. Saint-Raymond, The incompressible Navier-Stokes limit of the Boltzmann equation for hard cutoff potentials, J. Math. Pures Appl., (9)91, 508-552, 2009.

[17] H. Grad, Asymptotic theory of the Boltzmann equation, in Rarefied Gas Dynamics (Proc. 3rd Internat. Sympos., Palais de l'UNESCO, 1, 26-59, Paris, 1962. 
[18] D. Iftimie and F. Sueur, Viscous boundary layers for the Navier-Stokes equations with the Navier slip conditions, Arch. Ration. Mech. Anal., 199(1), 145-175, 2011.

[19] T. Kato, Remarks on zero viscosity limit for nonstationary Navier-Stokes flows with boundary, Seminar on Nonlinear Partial Differential Equations (Berkeley, Calif., 1983), 85-98, Math. Sci. Res. Inst. Publ., Springer, New York, 2, 1984.

[20] C.D. Levermore and N. Masmoudi, From the Boltzmann equation to an incompressible NavierStokes-Fourier system, Arch. Ration. Mech. Anal., 196, 753-809, 2010.

[21] J. Leray, Essai sur le mouvement d'un liquide visqueux emplissant l'espace, Acta Math., 63, 193-248, 1934.

[22] J.L. Lions, Quelques méthodes de résolution des problèmes aux limites non linéaires, Dunod, Paris, 2002.

[23] P.L. Lions, Mathematical Topics in Fluid Mechanics, Vol. 1: Incompressible Models, The Clarendon Press, Oxford University Press, New York, 1996.

[24] P.L. Lions and N. Masmoudi, From Boltzmann Equation to the Navier-Stokes and Euler Equations I, Archive Rat. Mech. Anal., 158, 173-193, 2001.

[25] P.L. Lions and N. Masmoudi, From Boltzmann Equation to the Navier-Stokes and Euler Equations II, Archive Rat. Mech. Anal., 158, 195-211, 2001.

[26] N. Masmoudi and L. Saint-Raymond, From the Boltzmann equation to the Stokes-Fourier system in a bounded domain, Commun. Pure Appl. Math., 56, 1263-1293, 2003.

[27] S. Mischler, Kinetic equations with Maxwell boundary conditions, Ann. Scient. Ec. Norm. Sup., 43, 719-760, 2010.

[28] L. Saint-Raymond, Du modèle BGK de l'équation de Boltzmann aux équations d'Euler des fluides incompressibles, Bull. Sci. Math., 126, 493-506, 2002.

[29] L. Saint-Raymond, Convergence of solutions to the Boltzmann equation in the incompressible Euler limit, Arch. Ration. Mech. Anal., 166, 47-80, 2003.

[30] L. Saint-Raymond, Hydrodynamic limits: Some improvements of the relative entropy method, Ann. Inst. H. Poincaré Anal. Non Linéaire, 26, 705-744, 2009.

[31] L. Saint-Raymond, Hydrodynamic Limits of the Boltzmann Equation, Lecture Notes in Math., Springer-Verlag, Berlin Heidelberg, 1971, 2009.

[32] V. Sheffer, An inviscid flow with compact support in space-time, J. Geom. Anal., 3, 343-401, 1993.

[33] A. Shnirelman, On the nonuniqueness of weak solution of the Euler equation, Commun. Pure Appl. Math., 50, 1261-1286, 1997.

[34] V.A. Solonnikov and V.E. Ščadilov, On a boundary value problem for a stationary system of Navier-Stokes equations, Proc. Steklov Inst. Math., 125, 186-199, 1973.

[35] Y. Sone, Molecular Gas Dynamics: Theory, Techniques and Applications, Birkhäuser, Boston, 2007.

[36] C. Villani, Limites hydrodynamiques de l'équation de Boltzmann (d'après C. Bardos, F. Golse, C. D. Levermore, P.L. Lions, N. Masmoudi, L. Saint-Raymond), [Hydrodynamic limits of the Boltzmann equation (following C. Bardos, F. Golse, C.D. Levermore, P.L. Lions, N. Masmoudi, L. Saint-Raymond)], French, Séminaire Bourbaki, Vol. 2000/2001. Astérisque, 893, 365-405, 282, 2002.

[37] Y. Xiao and Z. Xin, On the vanishing viscosity limit for the 3-D Navier-Stokes equations with a slip boundary condition, Commun. Pure Appl. Math., 60, 1027-1055, 2007.

[38] H.T. Yau, Relative entropy and hydrodynamics of Ginzburg-Landau models, Letters Math. Phys., 22, 63-80, 1991. 